RECEIVED 2009 MonTh XX; ACCEPTED 2009 Month XX

Preprint typeset using LTEX style emulateapj v. 11/10/09

\title{
THREE-DIMENSIONAL SIMULATIONS OF MIXING INSTABILITIES IN SUPERNOVA EXPLOSIONS
}

\author{
N.J. HAMMER ${ }^{1}$ H.-TH. JANKA, ${ }^{1}$ AND E. MÜLLER ${ }^{1}$ \\ Received 2009 Month XX; accepted 2009 Month XX
}

\begin{abstract}
We present the first three-dimensional (3D) simulations of the large-scale mixing that takes place in the shock-heated stellar layers ejected in the explosion of a $15.5 M_{\odot}$ blue supergiant star. The blast is initiated and powered by neutrino-energy deposition behind the stalled shock by means of choosing sufficiently high neutrino luminosities from the contracting, nascent neutron star, whose high-density core is excised and replaced by a retreating inner grid boundary. The outgoing supernova shock is followed beyond its breakout from the stellar surface more than two hours after the core collapse. Violent convective overturn in the postshock layer causes the explosion to start with significant large-scale asphericity, which acts as a trigger of the growth of Rayleigh-Taylor instabilities at the composition interfaces of the exploding star. Despite the absence of a strong Richtmyer-Meshkov instability at the $\mathrm{H} / \mathrm{He}$ interface, which only a largely deformed shock could instigate, deep inward mixing of hydrogen is found as well as fast-moving, metal-rich clumps penetrating with high velocities far into the hydrogen envelope of the star as observed, for example, in the case of Supernova 1987A. Also individual clumps containing a sizeable fraction of the ejected iron-group elements (up to several $10^{-3} M_{\odot}$ ) are obtained in some models. The metal core of the progenitor is partially turned over with nickel-dominated fingers overtaking oxygen-rich bullets and both nickel and oxygen moving well ahead of the material from the carbon layer. Comparing with corresponding two-dimensional (axially symmetric; 2D) calculations, we determine the growth of the Rayleigh-Taylor fingers to be faster, the deceleration of the dense metal-carrying clumps in the helium and hydrogen layers to be reduced, the asymptotic clump velocities in the hydrogen shell to be higher (up to $\sim 4500 \mathrm{~km} \mathrm{~s}^{-1}$ for the considered progenitor and an explosion energy of $10^{51} \mathrm{ergs}$, instead of $\lesssim 2000 \mathrm{~km} \mathrm{~s}^{-1}$ in $2 \mathrm{D}$ ), and the outward radial mixing of heavy elements and inward mixing of hydrogen to be more efficient in $3 \mathrm{D}$ than in $2 \mathrm{D}$. We present a simple argument that explains these results as a consequence of the different action of drag forces on moving objects in the two geometries.
\end{abstract}

Subject headings: hydrodynamics — instabilities — shock waves — supernovae: general

\section{INTRODUCTION}

Besides an experimental confirmation of a core collapse event through the detection of supernova neutrinos (Hirata et al. 1987), the second most important insight provided by observations of Supernova 1987A was the occurrence of large-scale non-radial flow and extensive mixing of chemical species in the envelope of the progenitor star during the explosion (see, e.g., Arnett et al. 1989). While SN 1987A still remains the most prominent and thoroughly-observed example, observations of many other core-collapse supernovae (SNe) have meanwhile provided ample evidence that largescale extensive mixing seems to occur generically in such events (see, e.g., Wang \& Wheeler 2008). In particular, the modeling of the light curve of SN 1987A using 1D radiation hydrodynamic calculations requires a large amount of mixing of $\mathrm{Ni}$ outward to the $\mathrm{H}-\mathrm{He}$ interface and of $\mathrm{H}$ inward into the He-core (Woosley 1988; Shigeyama \& Nomoto 1990; Blinnikov et al. 2000; Utrobin 2004). Moreover, the asymmetry of iron and nickel lines in SN 1987 can be explained, if $\mathrm{Ni}$ is concentrated into many high-velocity bullets (Spyromilio et al. 1990; Li et al. 1993). On the other hand, the Bochum event, i.e., the sudden development of fine-structure in the $H_{\alpha}$ line about two weeks after the explosion (Hanuschik et al. 1988), implies that a high velocity $(\sim 4700 \mathrm{~km} / \mathrm{s})$ clump of ${ }^{56} \mathrm{Ni}$ with a mass of $10^{-3} M_{\odot}$ was ejected into the far hemisphere of SN 1987 (Utrobin et al.

\footnotetext{
${ }^{1}$ Max-Planck-Institut für Astrophysik, Karl-Schwarzschild-Str. 1, D-85748 Garching, Germany; corresponding author: thj@mpagarching.mpg.de
}

1995).

Observations of near-infrared He I lines from Type II SNe between 50 and 100 days after core collapse imply mixing of ${ }^{56} \mathrm{Ni}$ into the hydrogen envelope (Fassia et al. 1998; Fassia \& Meikle 1999). These authors further conclude that those lines are formed in a clumpy environment. Dense knots, indications of ejecta shrapnels, and filaments seen in supernova remnants by HST in the visible (Blair et al. 2000; Fesen et al. 2006a) and by ROSAT, Chandra, and XMM in X-rays (Aschenbach et al. 1995; Hughes et al. 2000; Miceli et al. 2008) also provide strong evidence that mixing and perhaps even fragmentation is a common process in supernova explosions. Spectropolarimetric observations of Type II-P and Type IIn SNe at late epochs, when the hydrogen envelope starts thinning, reveal strong evidence for a globally highly aspherical distribution of the inner ejecta (Fassia et al. 1998; Leonard et al. 2001, 2006). Similar results are obtained from spectropolarimetric observations of Type Ib/c SNe (Kawabata et al. 2002; Wang et al. 2003; Maund et al. 2007; Modjaz et al. 2008; Wang \& Wheeler 2008). Finally, studying the nebula spectra of SN Ic 2002ap by the means of synthetic spectra, Mazzali et al. (2007) found evidence for an oxygen-rich inner core and ${ }^{56} \mathrm{Ni}$ at high velocities, suggesting a highly aspherical explosion especially in the inner parts.

Two-dimensional hydrodynamic simulations of non-radial flow and mixing in the stellar envelopes of core collapse supernova progenitors have been performed by several groups. The first simulations were started by artificially seeding Rayleigh-Taylor instabilities (RTIs) in the mantle and envelope of the progenitor and following their evolution until 
shock breakout from the stellar surface (Arnett et al. 1989; Fryxell et al. 1991; Müller et al.1991b. a; Hachisu et al. 1990; Herant \& Benz 1991; Hachisu et al. 1992; Herant \& Benz 1992). More recent simulations consistently connect the seed asymmetries arising from convective flow in the neutrinoheated bubble, and by the standing accretion shock instability (SASI) in the "supernova engine" during the first second of the explosion (see, e.g., Janka et al. 2007) to the later Rayleigh-Taylor and Richtmyer-Meshkov instabilities after shock passage through the outer stellar layers with application to SN 1987A (Kifonidis et al. 2003, 2006). Rayleigh-Taylor induced mixing and the amount of fallback occurring during artificially triggered (piston model), and initially spherically symmetric supernova explosions of zero- and solarmetallicity 15 and $25 M_{\odot}$ stars were studied by Joggerst et al. (2009). In all models considered by them the velocities of the heavy elements were less than those observed in SN 1987A. Couch et al. (2009) explored the observational characteristics of jet-driven supernovae in a red supergiant progenitor injecting $10^{51}$ ergs of kinetic energy and varying the characteristics of the assumed bipolar outflows (jets). Their simulations show the development of fluid instabilities that produce helium clumps in the hydrogen envelope, and nickel-rich jets that may account for the non-spherical excitation and substructure of spectral lines.

Only a few attempts to 3D simulations have been made up to now. Nagasawa et al. (1988) simulated the adiabatic point explosion of an $n=3$ polytrope using a 3D smoothed particle hydrodynamics (SPH) code, and claimed to have found pronounced Rayleigh-Taylor instabilities in that event. However, this claim was neither confirmed by the 3D simulations of Müller et al. (1989) using a 3D hydrodynamics code based on a high-resolution shock-capturing finite-volume method, nor by Benz \& Thielemann (1990) who also used a 3D SPH code. Both studies found only a weak instability, i.e., no extensive clumping and mixing, consistent with linear stability analysis. Subsequently, 3D simulations by Müller et al. (1991a) and Yamada et al. (1990) using relatively coarse resolution and/or considering only a wedge-shaped fraction of the star showed that seed perturbations grow strongly at the He$\mathrm{H}$ interface when a realistic presupernova star model is used instead of an $n=3$ polytrope. Later Kane et al. (2000) studied the difference in the growth of $2 \mathrm{D}$ versus $3 \mathrm{D}$ single-mode perturbations at the He-H and O-He interfaces of SN 1987A. They found that the $3 \mathrm{D}$ single-mode perturbation grows about $30 \%$ faster than a corresponding 2D one. Although the difference between 2D and 3D predicted by their simulations is not enough to account for the difference between observed ${ }^{56} \mathrm{Ni}$ velocities in SN 1987A and the results of previous 2D simulations of SN 1987A, their results suggest that non-radial flow and mixing in supernova envelopes in full 3D is noticeably different from that predicted by $2 \mathrm{D}$ simulations. This finding is also supported by laser experiments and comprehensive Rayleigh-Taylor growth simulations Anuchina et al. 2004; Cabot 2006; Remington et al.2006). The only other 3D simulations we are aware of are those published by Noro et al. (2002, 2004) in two short conference proceedings. These authors simulated the propagation of a 1D shock wave resulting from an artificially triggered spherical explosion of $10^{51} \mathrm{ergs}$ until it reaches the He-H interface of a SN 1987A progenitor model computed by Shigeyama \& Nomoto (1990). Then they perturbed the flow inside the shock radius and followed the growth of sinusoidal velocity perturbations with a 3D adap-

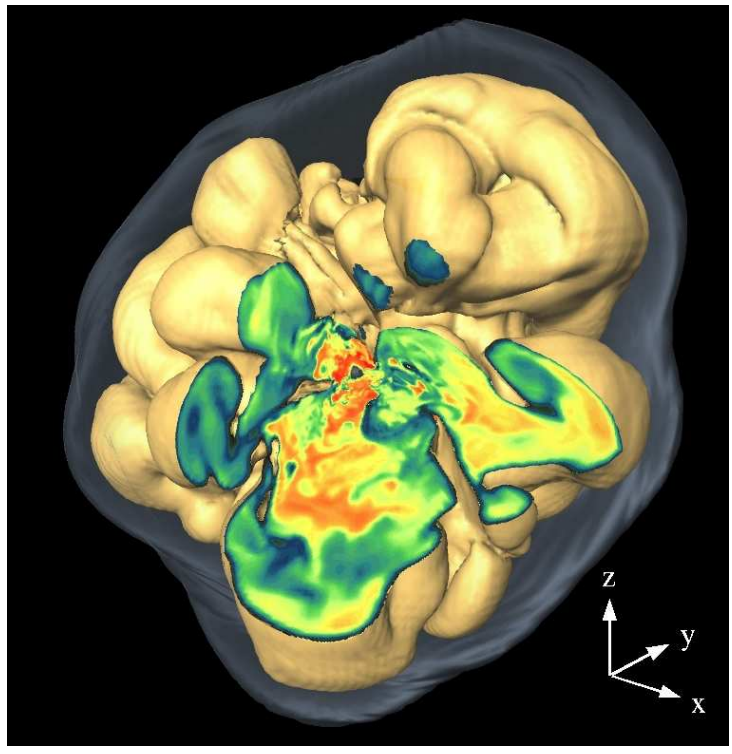

FIG. 1. - Three-dimensional, neutrino-driven explosion simulation at about $0.5 \mathrm{~s}$ after core bounce (Scheck 2007). The model is used as initial data for our studies. The outermost, bluish, nearly transparent surface is the supernova shock with an average radius of $1900 \mathrm{~km}$. The bright bumpy surface is the interface between rising, high-entropy neutrino-heated matter and lowerentropy, accreted post-shock gas. An octant is cut out to show the entropy distribution in the rising Rayleigh-Taylor bubbles and cooler downflows (the entropies per nucleon vary between about 10 and $21 k_{\mathrm{B}}$ from blue to yellow and red). The neutron star is visible as a dark grey surface near the center.

tive mesh hydro-code on a grid with an effective resolution of up to $4096^{3}$ zones. For an initial perturbation amplitude of $10 \%$, fingers of heavy elements penetrate into the hydrogen envelope with large velocities.

Here we simulate for the first time, starting with 3D models for the beginning of neutrino-powered explosions from Scheck (2007), the evolution of a supernova blast in 3D from the first hundreds of milliseconds to a time three hours later when the shock has broken out of a blue progenitor star. We find that not only the growth of Rayleigh-Taylor instabilities is different in 3D as concluded from other works (see above), but also that the propagation of "clumps" in 3D is different from the $2 \mathrm{D}$ case. In particular the latter effect is of large quantitative importance, because we demonstrate here that it determines the long-time evolution and in the end the extent of large-scale radial mixing in core-collapse supernovae. Quantitatively meaningful simulations of observable explosion asymmetries therefore require modeling in three spatial dimensions.

The paper is organized as follows. In Sect. 2 we describe the computational setup and the initial model used in our simulations. Our results are discussed in Sect. 3 where we particularly address the dynamical evolution and the radial mixing of chemical elements. In Sect. 4 we present a simple analytical model that explains the differences found for the clump propagation between 2D and 3D quite well. Finally, we summarize our findings in Sect. 5, and draw some conclusions.

\section{SIMULATION SETUP}

For our simulations we used the explicit finite-volume Eulerian multi-fluid hydrodynamics code, PROMETHEUS, developed by Bruce Fryxell and Ewald Müller (Fryxell et al. 1991; Müller et al. 1991ba). The code integrates the equations of multidimensional hydrodynamics to second order accuracy in space and time using the dimensional splitting 
approach of Strang (1968), the PPM reconstruction scheme (Colella \& Woodward 1984), and a Riemann solver for real gases according to Colella \& Glaz (1984). Multi-fluid flows (involving the advection of, e.g., several nuclear species; see below) are treated by means of a set of additional continuity equations using the Consistent Multi-fluid Advection (CMA) method of Plewa \& Müller (1999).

\subsection{Computational grid}

We use a computational grid in spherical polar coordinates consisting of $1200 \times 180 \times 360$ zones in $r, \theta$, and $\phi$-direction. The equidistant angular grid has a resolution of $0.935^{\circ}$ in $\theta$-direction and $1^{\circ}$ in $\phi$-direction, respectively, covering the whole sphere except for a (double) cone with a half opening angle of $5.8^{\circ}$ around the symmetry axis of the coordinate system (i.e., $0.0325 \pi \leq \theta \leq 0.9675 \pi$, and $0 \leq \phi \leq 2 \pi$ ). Reflective boundary conditions are imposed in $\theta$-direction, and periodic ones in $\phi$-direction. The clipping of the computational grid around the symmetry axis avoids a too restrictive CFL time step condition. We have not observed any numerical artifacts as consequences of this grid constraint.

The radial mesh is logarithmically spaced between a timedependent inner boundary, initially located at a radius of $200 \mathrm{~km}$, and a fixed outer boundary at $3.9 \times 10^{12} \mathrm{~cm}$. It has a maximum resolution of $2 \mathrm{~km}$ at the inner boundary, and a resolution of $4 \times 10^{10} \mathrm{~cm}$ at the outer one (corresponding to a roughly constant relative radial resolution $\Delta r / r \approx 1 \%$ ). We allow for free outflow at the outer boundary, and impose a reflective boundary condition at the inner edge of the radial grid.

During the simulation we move (approximately every 100th timestep) the inner radial boundary to larger and larger radii to relax the CFL condition. This cutting of the computational grid reduces the number of (the logarithmically spaced) radial zones from 1200 at the beginning to about 400 towards the end of the simulation, but involves only the innermost few percent of the initial envelope mass. We convinced ourselves by means of $2 \mathrm{D}$ test calculations that this removal of mass has no effect on the dynamics and mixing occurring at larger radii (the cut radius exceeds at no time a few percent of the radius of the Rayleigh-Taylor finger tips).

\subsection{Initial model}

The initial model used for our 3D simulations is based on 3D hydrodynamic explosion models of Scheck (2007), who followed the onset and early development of neutrino-driven explosions in 3D with the same code, numerical setup, and input physics as described in all details for 1D and 2D simulations in Scheck et al. (2006). These runs were started from 1D stellar collapse models a few milliseconds after core bounce. The explosion was initiated and powered by the energy input of neutrinos in the postshock layer, where vigorous convective activity was present and supported the revival of the stalled prompt shock. A defined value of the explosion energy was obtained by suitably choosing the magnitude of the neutrino luminosities that were imposed at the retreating inner grid boundary, which replaced the excised, contracting dense core of the forming neutron star.

The stellar progenitor considered here is a spherically symmetric model of a $15.5 M_{\odot}$ blue supergiant with a radius of $4 \times 10^{12} \mathrm{~cm}$ (Woosley et al. 1988, Woosley, private communication; see also Kifonidis et al. 2003). Since the models of Scheck (2007) included only the central part of the star out to the middle of the $\mathrm{C} / \mathrm{O}$ shell at $r=1.7 \times 10^{9} \mathrm{~cm}$, the envelope of the progenitor had to be added for the long-time simulations presented here.

The $15.5 M_{\odot}$ blue supergiant stellar progenitor model was evolved by Woosley et al. (1988) using a nuclear reaction network including a large set of nuclei. However, Scheck (2007) did not consider nuclear burning, and he included only a small number of nuclear species ( $p, n, \alpha$, and ${ }^{54} \mathrm{Mn}$ representing the heavy element fraction) in their simulations to save computational costs. For our initial model we kept the nuclear composition of the 3D explosion model of Scheck (2007) inside the shock radius, redefining ${ }^{54} \mathrm{Mn}$ into ${ }^{56} \mathrm{Ni}$. The nuclear composition at radii greater than the shock radius was taken from the $15.5 M_{\odot}$ progenitor model.

Scheck (2007) simulated one 3D hydrodynamic explosion model with $2^{\circ}$ angular resolution, which reached a final time of $0.58 \mathrm{~s}$ after bounce, and another one with $3^{\circ}$ angular resolution, which covered an evolution time of $1 \mathrm{~s}$ after core bounce. Although both models were exploited for our study, we report here only on simulations using the former model that according to the data of Scheck (2007) has an (unsaturated) explosion energy of $0.6 \times 10^{51} \mathrm{erg}$. The deformation of the shock front at $t \sim 0.5 \mathrm{~s}$ after core bounce and the asymmetry of the entropy distribution behind the shock in the form of rising, inflating hot plumes and infalling, narrow, cool downflows are shown in Fig. 1. The structure of the explosion at this time looks very similar to comparable results by Fryer \& Warren (2002). Besides following the shock's propagation through the stellar envelope of this initial model with our 3D code, we also performed a 3D simulation where we artificially increased the explosion energy of this initial model to $1.0 \times 10^{51} \mathrm{erg}$ by extending the thermal energy input as accomplishable by ongoing neutrino heating.

\subsection{Equation of state}

We used a tabulated EOS (Timmes \& Swesty 2000) that considers contributions of an arbitrarily degenerate and relativistic electron-positron gas, of a photon gas, and of a set of ideal Boltzmann gases, consisting of the eight nuclear species ( $n, p, \alpha,{ }^{12} \mathrm{C},{ }^{16} \mathrm{O},{ }^{20} \mathrm{Ne},{ }^{24} \mathrm{Mg}$, and ${ }^{56} \mathrm{Ni}$ ) included in our initial model. Note that the pre-shock layers contained only a small region of silicon at the time the explosion model of Scheck (2007) was adopted as initial data for our long-time supernova runs. Since nuclear burning was not taken into account in our simulations, but it is possible that this silicon will be explosively burned to nickel, we lumped the small amount of silicon and the iron-group elements in the postshock layer together to what we followed as ${ }^{56} \mathrm{Ni}$ in our hydrodynamic calculations.

\subsection{Simulation runs}

We performed a set of thirteen 2D and three 3D simulations and compared the results obtained in three dimensions to those of the corresponding 2D models. In this paper we focus our discussion on one 3D model that has an explosion energy of $10^{51}$ ergs. The main findings for this particular 3D model are qualitatively similar to those we obtained for less energetic 3D explosions, but the resulting maximum values for the asymptotic clump velocities vary with the explosion energy roughly as $v_{\text {clump }} \propto \sqrt{E_{\text {exp }}}$.

The 2D simulations reported here were started from different meridional slices of the 3D initial explosion model. This 
ensures as much similarity of the initial conditions as possible and the same numerical resolution for the 2D and 3D runs. But the differences between the chosen slices give rise to some variation of the initial asymmetry and explosion energy among the various 2D models, which manifests itself both in different clump structures and velocities, and the amount of mixing in the stellar envelope.

A set of 13 meridional 2D slices was placed at azimuthal angles of 4, 36, 76, 108, 128, 148, 180, 220, 228, 252, 292, 324, and 360 degrees. Three of these slices (at 128, 220 , and 228 degrees) were chosen at locations where the growth of Rayleigh-Taylor fingers was observed to be particularly strong in $3 \mathrm{D}$. This guaranteed that such regions were not missed by our comparison of $2 \mathrm{D}$ and $3 \mathrm{D}$ calculations. It turned out that the largest 3D Rayleigh-Taylor structures at late stages develop in the directions of the biggest initial buoyant bubbles, expanding towards the bottom edge and upper right corner (at six o'clock and one o'clock, respectively) of Fig. 1

Both in the 3D and 2D simulations we neglected the influence of gravity on the motion of the ejecta. While this has no important impact on the dynamics of the expanding ejecta, the amount of fallback is underestimated. However, that way we could avoid the accumulation of mass near the inner (reflecting) radial boundary which would have implied a considerably more restrictive CFL condition.

\section{RESULTS}

\subsection{Dynamical evolution}

The dynamical evolution of the explosion after its launch by neutrino energy deposition and the growth of instabilities in the considered $15.5 M_{\odot}$ progenitor were described in much detail by Kifonidis et al. (2003). It was shown there that the asymmetries associated with the convective activity developing in the postshock region during the neutrino-heating phase act as seeds of secondary Rayleigh-Taylor instabilities at the composition interfaces of the exploding star. At about $100 \mathrm{~s}$ dense Rayleigh-Taylor fingers containing the metals $(\mathrm{C}, \mathrm{O}, \mathrm{Si}$, iron-group elements) have grown out of a compressed shell of matter left behind by the shock passing through the $\mathrm{Si} / \mathrm{O}$ and $(\mathrm{C}+\mathrm{O}) / \mathrm{He}$ interfaces. These fingers grow quickly in length and while extending into the helium shell, fragment into ballistically moving clumps and filaments that propagate faster than the expansion of their environment.

While for a 2D model with explosion energy around $1.8 \times 10^{51} \mathrm{erg}$ the $\mathrm{Si}$ and $\mathrm{Ni}$ containing structures still move with nearly $4000 \mathrm{~km} \mathrm{~s}^{-1}$ (oxygen has velocities up to even $5000 \mathrm{~km} \mathrm{~s}^{-1}$ ) at $300 \mathrm{~s}$, a strong deceleration occurs when the metal clumps enter the relatively dense He-shell that forms after the shock passage through the $\mathrm{He} / \mathrm{H}$ interface. At $t \gtrsim$ $10,000 \mathrm{~s}$ the metal carrying clumps have dissipated their excess kinetic energy and propagate with the same speed as the helium material in their surroundings. In the presence of a hydrogen envelope and the corresponding deceleration of the shock as it propagates through the inner regions of the hydrogen layer (in which case the helium "wall" below the $\mathrm{He} / \mathrm{H}$ interface builds up), Kifonidis et al. (2003) could not observe any metal clumps that achieve to penetrate into the hydrogen envelope, in contrast to what was observed in the case of SN 1987A.

The 2D calculations performed in the course of the present work confirm these findings (when the velocities are appropriately rescaled with $\sqrt{E_{\exp }}$ to account for the lower explo- sion energies of $E_{\exp } \lesssim 10^{51}$ erg considered here compared to roughly twice this value employed by Kifonidis et al. 2003). The evolution as well as the final results for the mass distributions of different chemical elements in velocity space and mass space are in good quantitative agreement with the models of Kifonidis et al. (2003), although the latter had much superior numerical resolution because of the use of a sophisticated adaptive mesh refinement algorithm. We are therefore confident that at least with respect to gross (quasi-1D) features, the results presented here are numerically converged, even if many of the structural details, including plumes and bullets, may not be numerically converged.

Kifonidis et al. (2006), investigating explosions also with $E_{\text {exp }} \sim 2 \times 10^{51} \mathrm{erg}$, proposed a cure of the metal-H mixing problem by invoking a sufficiently large dipolar or quadrupolar asymmetry of the beginning explosion with a globally aspherical shock wave. This was motivated by recent hydrodynamical studies that show that large-amplitude, low- $\ell$ mode oscillations ( $\ell$ defining the order of an expansion in spherical harmonics) due to the standing accretion shock instability (SASI) can be an important ingredient in the explosion mechanism and in the sequence of processes that leads to observed asymmetries of supernova explosions and neutron star kicks (see e.g., Blondin et al. 2003; Blondin \& Mezzacappa 2007; Scheck et al. 2004, 2006; Scheck 2007; Scheck et al. 2008; Burrows et al. 2006, 2007; Marek \& Janka 2009). This on the one hand led to higher initial maximum velocities of the metal-rich clumps and thus their faster propagation through the He core on a timescale shorter than the build-up of the thick He "wall" that prevented their penetration into the hydrogen shell in the older calculations. On the other hand it triggered the growth of Richtmyer-Meshkov instability (RMI) at the $\mathrm{He} / \mathrm{H}$ interface due to the deposition of vorticity by the shock. This led to strong mixing of hydrogen inward in mass space and down to low velocities, an effect that has turned out to be necessary to explain the shape of the light curve maximum in the case of SN 1987A (Utrobin 2004).

These conclusions, however, were based on 2D models. Therefore the question remained whether 3D might yield differences and whether also in 3D one would have to advocate a pronounced global low- $\ell$ mode asphericity of the beginning explosion as an explanation of the high velocities $\left(\gtrsim 3000 \mathrm{~km} \mathrm{~s}^{-1}\right)$ of nickel clumps in the hydrogen shell and of the strong inward mixing of hydrogen observed in SN 1987A. The 3D simulations performed in the present work demonstrate that this is not the case and the mentioned observational features can be accounted for by 3D models even with explosion energies around $10^{51} \mathrm{ergs}$ for the considered progenitor star.

In order to quantify the global shock deformation of our $3 \mathrm{D}$ initial models compared to the $2 \mathrm{D}$ cases investigated by Kifonidis et al. (2006), we did not perform an analysis in terms of an expansion in spherical harmonics modes. Instead, sizeable differences become obvious already when a triaxial ellipsoid is constructed as envelope of the bumpy shock surface obtained by Scheck (2007) such that the major axis of the ellipsoid coincides with the direction of the strongest shock expansion. While the 3D model used for the calculations of the present paper has an axis ratio of 1.04:1.02:1.00 at $t=0.58 \mathrm{~s}$ after bounce - another $3 \mathrm{D}$ explosion run of Scheck (2007) yields 1.16:1.06:1.00 at $t=1 \mathrm{~s}-$, the shocks of the 2D configurations studied by Kifonidis et al. (2006) typically had much larger axis ratios of about 1.5:1.0. 

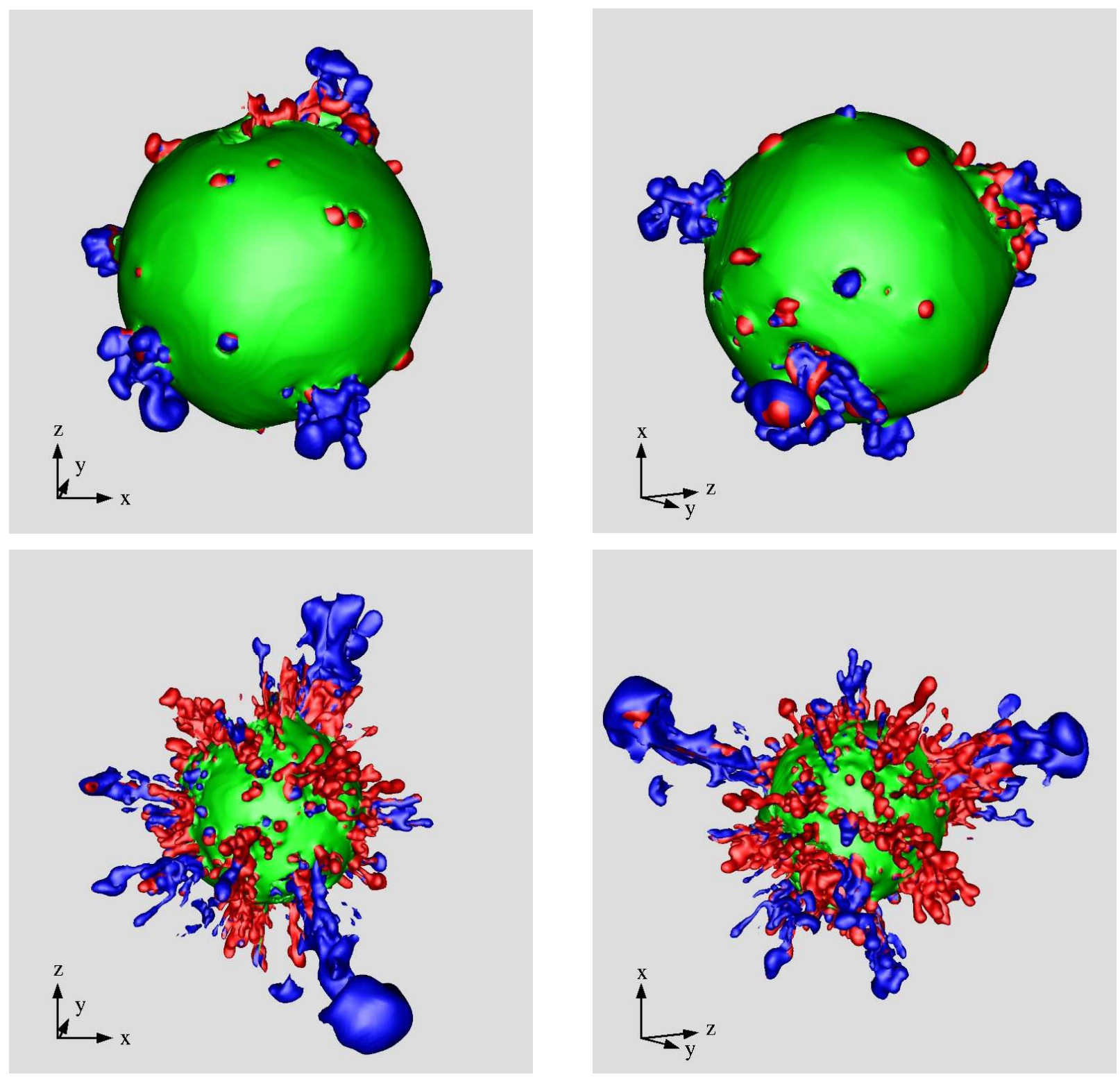

FIG. 2.- Surfaces of the radially outermost locations with constant mass fractions of $\sim 3 \%$ for carbon (green), and oxygen (red), and of $\sim 7 \%$ for nickel (blue). The upper two panels show the directional asymmetries from two different viewing directions at $350 \mathrm{~s}$ after core bounce when the metal clumps begin to enter the helium layer of the star. The lower two panels display the hydrodynamic instabilities at about $9000 \mathrm{~s}$ shortly after the supernova shock has broken out of the stellar surface. The side length of the upper panels is about $5 \times 10^{11} \mathrm{~cm}$, of the lower panels $7.5 \times 10^{12} \mathrm{~cm}$.

The deformed shock causes strong Richtmyer-Meshkov instability (RMI) to develop at the $(\mathrm{C}+\mathrm{O}) / \mathrm{He}$ and $\mathrm{He} / \mathrm{H}$ interfaces in the 2D models discussed here. Compared to the runs of Kifonidis et al. (2006), however, the smaller asphericity of the shock leads to a much slower growth of the instability. In our $2 \mathrm{D}$ runs the corresponding vortices at the $\mathrm{He} / \mathrm{H}$ boundary at $13000 \mathrm{~s}$ have reached a size relative to the average interface radius similar to what was observed at $3000 \mathrm{~s}$ in the models of Kifonidis et al. (2006). We note in passing that apart from resolution-dependent fine structure, the results of the latter paper could be very well reproduced concerning the RMI vortex shape, size, and locations by simulations performed for the same initial conditions but with the grid setup and resolution used in the present work (see Hammer (2009)). The growth of the RMI observed in average cases of our $2 \mathrm{D}$ calculations with only weak low- $\ell$ mode shock deformation - in agreement with analytic growth rate estimates; Hammer (2009) - is much too slow to produce any significant extent of hydrogen-helium mixing so that the final outcome of our 2D models basically confirms the findings of Kifonidis et al. (2003) In the 3D models RMI distortions can be seen at the $(\mathrm{C}+\mathrm{O}) / \mathrm{He}$ interface and are likely to contribute to the turbulent mixing of the metal core with the helium shell of the exploding star. At the $\mathrm{H} / \mathrm{He}$ interface, however, where the shock is very close to being spherical, no clear RMI activity becomes visible before it is penetrated by fast, metal-carrying clumps that have been able to pass through the helium layer

${ }^{2}$ However, there is a considerable spread of the 2D results depending on the choice of the meridional plane of the 2D slice and the variation of the conditions between the different planes. Slices with somewhat larger initial shock deformation show stronger late mixing into the hydrogen shell than the more typical "average" 2D slices (see Fig. 8. 


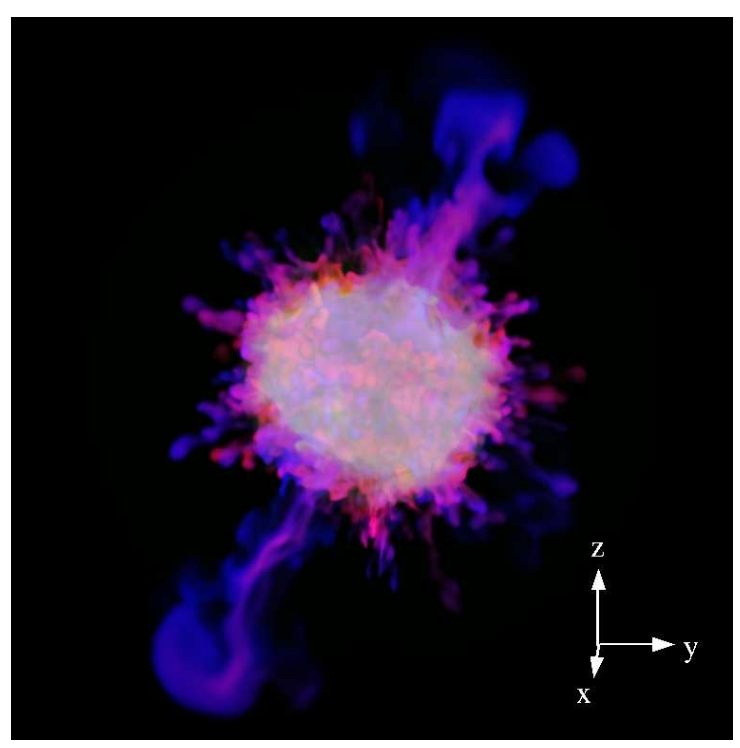

FIG. 3. - Volume rendered image of the element distribution at about $9000 \mathrm{~s}$ (corresponding to the lower two panels in Fig. 2 but displayed for yet another viewing angle). The blue structures contain a dominant mass fraction of nickel, the red fingers mostly oxygen, and green is associated with carbon. A mixture of nickel and oxygen appears in pink. Note that the green visible in Fig. 2 has been subsumed into the white glow due to the contamination with other colors as a consequence of the volume rendering and not of true mixing. The side length of the displayed volume is about $7.5 \times 10^{12} \mathrm{~cm}$. It is remarkable that the two long Rayleigh-Taylor fingers from this perspective create the impression of a jet and an anti-jet, although no jet-creating mechanism was in action at the center of the explosion and the long Rayleigh-Taylor structures do actually not point into exactly opposite (i.e., antiparallel) directions (cf. Fig. 2].

with still high velocities.

\subsection{Radial element mixing}

Fig. 2 displays the development of these fast-moving clumps during our 3D explosion run by showing surfaces of constant mass fractions of carbon, oxygen, and nickel for two different viewing directions and two different times (350s and $\sim 9000 \mathrm{~s}$ after core bounce). Figure 3 provides a volume-rendered image of the composition distribution at the later time, while Fig. 4 gives composition information on cut planes through the mixed stellar core and some of the major plumes of different types. Finally, Figs. 6 and 7 present normalized mass distributions of various nuclear species in the radial-velocity and enclosed-mass space for our 3D simulation compared to an "average" $2 \mathrm{D}$ result at several times after core bounce, and Fig. 8 provides information for the spread of the hydrogen and nickel mass distributions in our set of 2D runs at the end of the simulations.

We stress that what we denote as "nickel" here and in the following actually includes the contributions of silicon and of the iron-group elements from the postshock region. This makes sense because the shock front in the initial model has nearly reached the oxygen layer (which implies that only a very small fraction of the original silicon is left) and because we do not account for nuclear burning in our simulations (for which reason no silicon is produced lateron); see Sect. 2.3. In the $3 \mathrm{D}$ model the total mass of free neutrons is $6 \times 10^{-4} M_{\odot}$, of hydrogen $8.16 M_{\odot}$, of helium $5.40 M_{\odot}$, of carbon $0.12 M_{\odot}$, of oxygen $0.20 M_{\odot}$, of neon $0.048 M_{\odot}$, of magnesium $0.005 M_{\odot}$, and of "nickel" $0.212 M_{\odot}$. About $1.35 M_{\odot}$ of the $15.5 M_{\odot}$ progenitor are thus absorbed into the neutron star. In the 2D runs based on selected meridional slices, the ejecta masses of the different nuclear species can differ insignificantly from the listed numbers.

Figure 2 shows that at 350 s oxygen and nickel rich, dense fingers, some of which display the typical mushroom shape of Rayleigh-Taylor structures, penetrate through the outer boundary of the carbon layer of the exploding star. The corresponding ${ }^{12} \mathrm{C}$ surface is defined by a mass fraction of $3 \%$ (note that also exterior to this surface, in the surrounding helium and hydrogen layers, a small but non-zero mass fraction of carbon is still present and representative of the heavy elements that define the metallicity of the progenitor star). The most prominent features at that time have been seeded by the biggest high-entropy bubbles visible in Fig. 1 and develop to the largest mushrooms visible more than 8000 s later.

The chemical composition of the various kinds of extended plumes in this late stage is very interesting. All of them contain high mass fractions of hydrogen and helium, which account for about 50 to 70 percent of their mass with spatial variations in individual filaments. The dominant heavy species, however, differs between different structures. The largest mushrooms carry predominantly nickel and may contain a narrow spine with oxygen. In contrast, many of the far more numerous, less extended and more narrow fingers are composed of a mixture of nickel and oxygen. Also clumps with a dominant mass fraction of oxygen can be identified. More useful for such an analysis than the surface images of Fig. 2 is the visualization by volume-rendering techniques in Fig. 3. While the surfaces of constant mass fraction can be misleading, because their shape depends strongly on the chosen value and no information about the interior composition is provided, the ray-tracing image shows that the largest Rayleigh-Taylor mushrooms carry mostly nickel, the majority of the more extended fingers are composed of a mix of elements, and only some clumpy, relatively compact, knotty features (clearly visible along the edge of the pastel-colored and whitish glowing core in Fig. 3) contain a dominant fraction of oxygen. This is confirmed by the planar cuts through two typical Rayleigh-Taylor structures in Fig. 4 The upper left panel of this figure, in which a 2D cut through the whole core is displayed, demonstrates that on the global scale there are extended and separated regions where different species are the most abundant chemical constituent. In the core the hydrogen and helium admixture is much smaller than in the extended fingers and plumes. Except in some smaller "bubbles", where $\mathrm{H}+\mathrm{He}$ dominate, heavies contribute $50-80 \%$, in some clumps even more than $90 \%$, of the core composition.

The time sequences of the normalized mass distributions of the nuclear species in the radial-velocity and enclosed-mass space 3 of Figs. 6 and 7 visualize the gradual acceleration of the outer $\mathrm{He}$ and $\mathrm{H}$ layers of the exploding star by the outgoing shock front. Simultaneously, the initially fast metals of the core penetrate into the outer composition layers and are decelerated. Both the deceleration and the mixing depend crucially on the hydrodynamic instabilities that develop at the composition interfaces, seeded by the initial explosion asymmetries. At 350s the helium "wall" can be discerned as the peak of the distribution between $3500 \mathrm{~km} \mathrm{~s}^{-1}$ and $4500 \mathrm{~km} \mathrm{~s}^{-1}$ at an enclosed mass between $\sim 2.5 M_{\odot}$ and $\sim 4.5 M_{\odot}$. From a comparison of the plots for 2D and 3D it is obvious that nickel

3 The mass distribution of each nuclear species is normalized such that the radial integration in velocity or enclosed-mass space gives unity. Thereby the mass $\Delta M_{i}$ of an element $i$ in a velocity or mass bin is divided by the total mass $M_{i}$ of this element on the grid at the time of evaluation. 

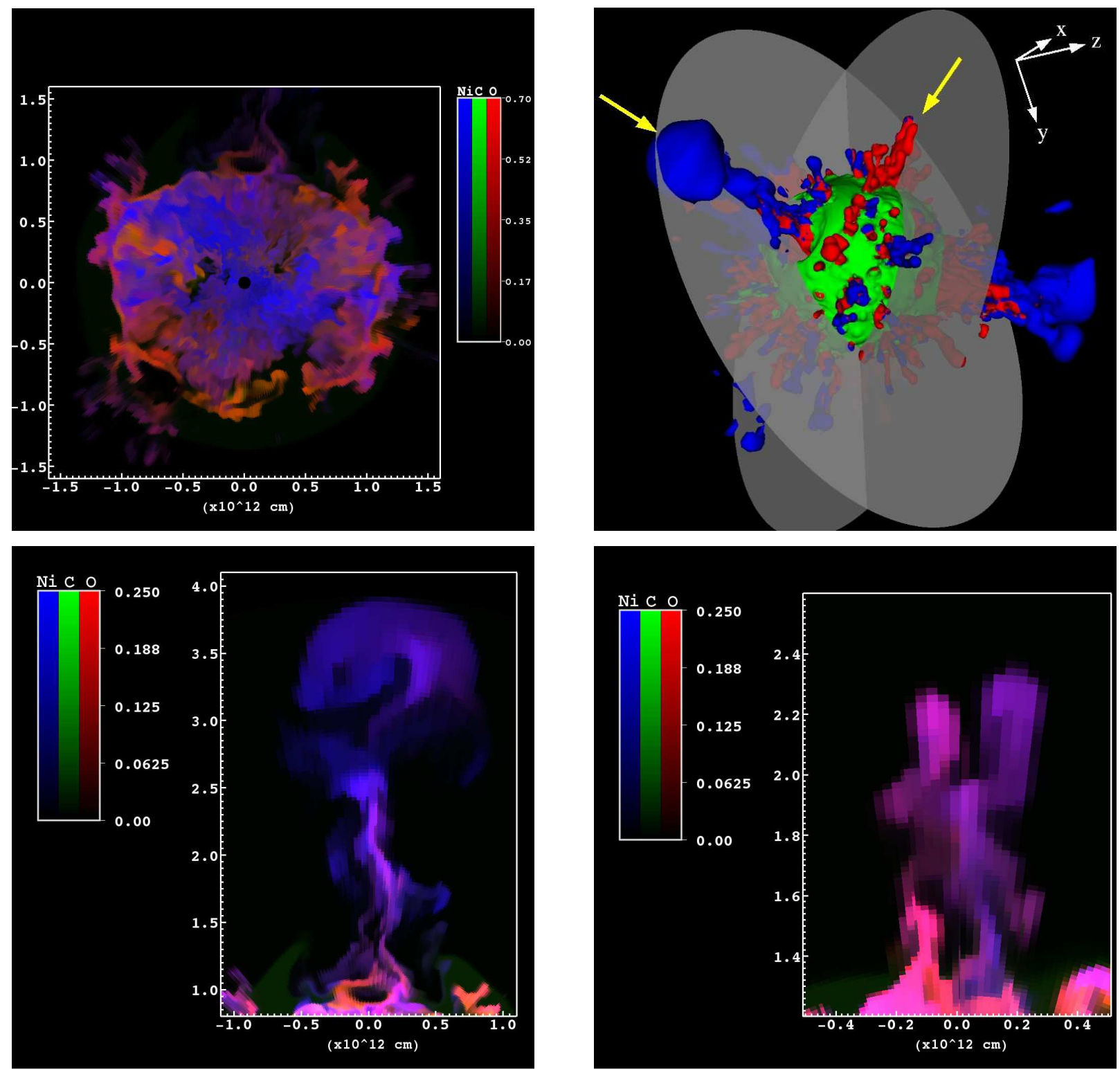

FIG. 4.- Composition information on 2D cuts through the 3D model structure. Blue corresponds to nickel, red to oxygen, green to carbon. Note that for better visibility of the dynamical variation, different color ranges were chosen for the mass fractions in the global-view image (top left) and in the local zooms (bottom). Upper left: Mass fractions of $\mathrm{Ni}, \mathrm{O}$, and $\mathrm{C}$ color coded in a cross sectional plane through the mixed core. The cut plane is indicated in the upper right panel and crosses the big mushroom that is enlarged in the lower left panel (this feature grows out of the core at the 12:00 o'clock position), but the basic features of the core structure do not depend much on the chosen location of the plane through the grid origin. Upper right: Cutting planes through two representative Rayleigh-Taylor structures, the big mushroom growing towards 10 o'clock, and the multi-finger feature extending towards 12:30 o'clock. Both features are marked by thick yellow arrows. Lower left: Composition in the cut plane through the big Rayleigh-Taylor mushroom. Lower right: Composition in the cut plane through the multi-finger region. All extended plumes and fingers carry a mix of heavy elements with different mass fractions and relative abundances, but also large amounts of hydrogen and helium (between $\sim 50$ and $\sim 70 \%$ of the mass with considerable spatial variations inside individual structures).

and oxygen experience a much stronger deceleration during the subsequent evolution and far less efficient mixing into the hydrogen envelope in the $2 \mathrm{D}$ case (the plane of the meridional cut considered in Figs. 6 and 7 is displayed in Fig. 5.4.

\footnotetext{
4 The set of meridional slices of the 3D initial data that we used for 2D simulations exhibits variations at a certain level with respect to gas energies and asymmetries of the mass distribution. This leads to corresponding differences in the development of nonradial hydrodynamic instabilities in the outer stellar layers. The case picked for Figs. 6 and 7 can be considered as "average" concerning metal clump velocities and mixing, but some of the 2D slices also yield slightly more extreme results (cf. Fig. 8). In particular, the set of slicing directions was chosen such that 3D regions with particularly strong growth of Rayleigh-Taylor fingers were not missed (Sect. 2.4.
}

Since neon and magnesium are carbon-burning ashes and well mixed with oxygen in the oxygen-layer of the progenitor star, the distributions of these three elements closely match each other during the mixing evolution and can be hardly distinguished on plots. We therefore have combined them into one line in Figs. 6 and 7

A closer inspection of Figs. 6 and 7 reveals that the metal distributions in both 2D and 3D models peak at the location of the metal core (enclosed mass $M_{r} \lesssim 2.5 M_{\odot}$ ) with similar velocities (around 2000-3000 $\mathrm{km} \mathrm{s}^{-1}$ at $350 \mathrm{~s}$ and $1000 \mathrm{~s}$, around $1500-2500 \mathrm{~km} \mathrm{~s}^{-1}$ at $2600 \mathrm{~s}$, and around $1000 \mathrm{~km} \mathrm{~s}^{-1}$ at 9000 s). In 3D, however, the nickel and oxygen distributions 


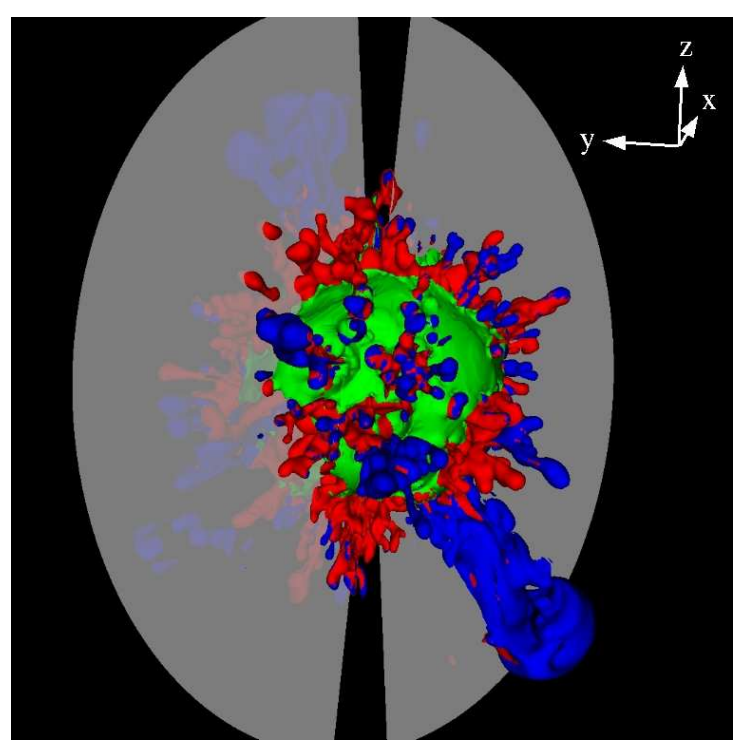

FIG. 5.- Plane of the meridional cut at an azimuthal angle of 148 degrees, which was the basis of the 2D simulation whose results are plotted in comparison to 3D in Figs. 6and77 Note that we chose a "typical" (average) direction for the cut instead of an extreme one, while the variation of the $2 \mathrm{D}$ results in different directions is indicated in Fig. 8

possess much more massive and wider high-velocity tails up to velocities of more than $6000 \mathrm{~km} \mathrm{~s}^{-1}$ at $350 \mathrm{~s}$. In the long run this is causal for the large discrepancies of the radial composition mixing. Two effects appear to contribute to the formation and continued presence of these high-velocity tails:

(i) In 3D the Rayleigh-Taylor instabilities at the composition interfaces (in particular the $(\mathrm{C}+\mathrm{O}) / \mathrm{He}$ boundary) grow more rapidly. This leads to the formation of extremely fast (mostly nickel and less oxygen) clumps with velocities in excess of $5000 \mathrm{~km} \mathrm{~s}^{-1}$ at $350 \mathrm{~s}$ (upper right panel of Fig. 6). These dense bullets penetrate deep into the hydrogen layer even before the helium wall has formed (see the upper two right panels in Fig. 7). The more rapid growth of the Rayleigh-Taylor instabilities also seems to be the reason for the efficient mixing of hydrogen into the metal core, which is clearly different from the $2 \mathrm{D}$ case already at $\sim 1000$ s.

(ii) A larger fraction of the nickel and oxygen with a velocity of $\sim 4000 \mathrm{~km} \mathrm{~s}^{-1}$ at $350 \mathrm{~s}$, which is in the ballpark of the maximum nickel and oxygen velocities of the $2 \mathrm{D}$ models, experiences less velocity reduction in the $3 \mathrm{D}$ simulation. This is obviously linked to a different propagation and deceleration of the dense metal-containing clumps as they move quasi-ballistically through the helium shell and inner part of the hydrogen envelope under the influence of drag forces exerted by the surrounding medium. While the fastest bullets with a speed of $5000-6000 \mathrm{~km} \mathrm{~s}^{-1}$ at $350 \mathrm{~s}$ still move with 3000 $4500 \mathrm{~km} \mathrm{~s}^{-1}$ at $t>2600 \mathrm{~s}$, the clumps with initially $\sim 4000 \mathrm{~km} \mathrm{~s}^{-1}$ are decelerated to $2000-3000 \mathrm{~km} \mathrm{~s}^{-1}$. In contrast, in 2D none of the material of the metal core has retained velocities of more than $2000 \mathrm{~km} \mathrm{~s}^{-1}$ at the time when the shock reaches the stellar surface $(t \sim$ 8000 s). We will return to a detailed discussion of this very interesting phenomenon in Sect. 4 where we will present simple analytic arguments for an explanation.
In Fig. 8 the normalized mass distributions of hydrogen and nickel in the radial velocity space are plotted at $t \sim 9000 \mathrm{~s}$ shortly after the shock breakout from the stellar surface (when the expansion is close to become homologous) for our 3D run in comparison to the compilation of results obtained for all 2D simulations with different chosen meridional slices. While the maximum hydrogen velocities are very similar in $2 \mathrm{D}$ and $3 \mathrm{D}$ and reach up to about $6000 \mathrm{~km} \mathrm{~s}^{-1}$, a clearly bigger hydrogen mass attains the lowest velocities (below $1000 \mathrm{~km} \mathrm{~s}^{-1}$ ) in the 3D case. Correspondingly, a small fraction of the hydrogen (some hundredths of a solar mass) is mixed deep into the metal core of the star to regions with enclosed masses of $M(r) \lesssim 2 M_{\odot}$ (see also the right panels of Fig. 7). These findings are very similar to the results obtained in $2 \mathrm{D}$ by Kifonidis et al. (2006; cf. figures 6, 8, and 9 there), who considered cases with largely aspherical beginning of the explosion as discussed in Sect. 3.1. In 3D similarly strong inward mixing of hydrogen does not require a very pronounced global asymmetry of the early blast. Richtmyer-Meshkov instability at the shock-distorted $\mathrm{He} / \mathrm{H}$ composition interface plays no crucial role in our 3D simulations, because the outgoing supernova shock is significantly less deformed than in the $2 \mathrm{D}$ models discussed by Kifonidis et al. (2006), see Sect.3.1.

Even more impressive than the hydrogen differences is the large discrepancy of 2D and 3D with respect to the nickel distribution in velocity space and the maximum nickel velocities. While in 2D even the fastest nickel clumps move with just $2200 \mathrm{~km} \mathrm{~s}^{-1}$ at $t \sim 9000 \mathrm{~s}$ (Fig. 8), a large fraction of the nickel retains velocities of more than $3000 \mathrm{~km} \mathrm{~s}^{-1}$ and some even more than $4000 \mathrm{~km} \mathrm{~s}^{-1}$ in 3D (Fig. 8). From Fig. 6 we can conclude that similarly large $2 \mathrm{D}$ vs. 3D differences are obtained for the oxygen distribution in velocity space, whereas the hydrogen and helium distributions of the 2D case are close to the results of the $3 \mathrm{D}$ run (except for the lowvelocity tail of the hydrogen distribution mentioned above), and the carbon distribution exhibits slight differences only between about $1000 \mathrm{~km} \mathrm{~s}^{-1}$ and $2500 \mathrm{~km} \mathrm{~s}^{-1}$. Interestingly, a significantly larger fraction of the nickel than of the oxygen propagates with the highest speeds of clumps. This is compatible with the fact that the metal content of the biggest and most extended Rayleigh-Taylor structures in Fig. [2] is dominated by nickel, while oxygen is concentrated mostly in thin, shorter cores of the mushrooms (see Fig. 4).

It should also be noted that the spread of the maximum nickel velocities obtained in the set of $2 \mathrm{D}$ runs in Fig. 8 is considerably smaller than the corresponding spread of the 2D results in the mass space. The maximum Ni velocities vary only between about $1700 \mathrm{~km} \mathrm{~s}^{-1}$ for average and $2200 \mathrm{~km} \mathrm{~s}^{-1}$ for extreme $2 \mathrm{D}$ cases (in which the meridional slices were placed at the locations of the biggest nickel mushrooms in 3D). In the former models nickel is transported to slightly more than $5 M_{\odot}$, whereas in the latter ones some of the nickel gets mixed out to an enclosed mass of even $8-9 M_{\odot}$. In all the cases, however, the mixing of iron-group elements into the hydrogen layer is much less strong in 2D than in $3 \mathrm{D}$.

Figure 7 showing an average 2D run, confirms these findings. In the latest stages nickel and oxygen can be observed at an enclosed mass of about $5 M_{\odot}$ (in rough agreement with the results of Kifonidis et al. (2003), see their fig. 15), which is close to the base of the hydrogen envelopes. In typical 2D

5 The slightly deeper intrusion of metals into hydrogen in our models can be understood as a consequence of the lower explosion energy, which leads 

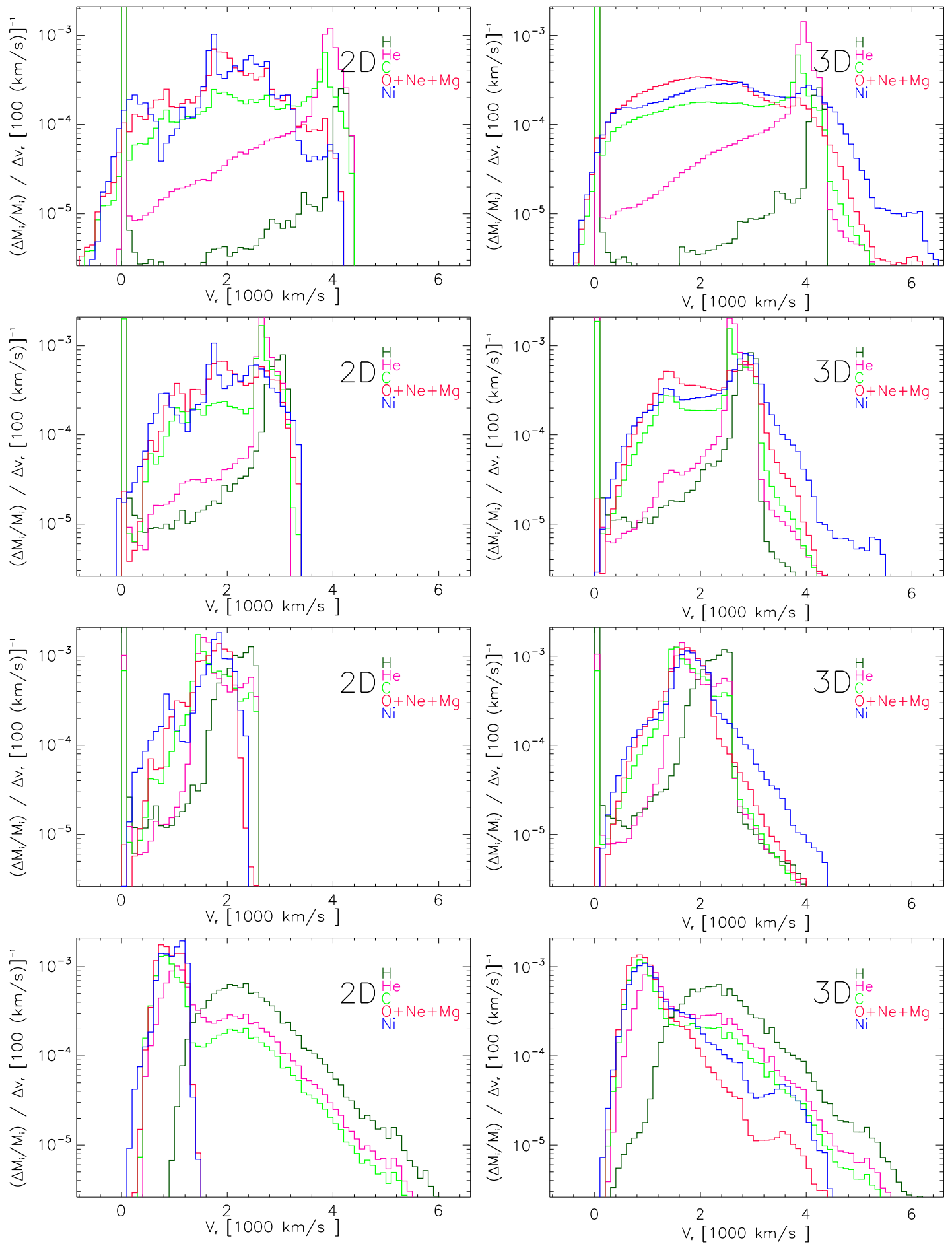

FIG. 6.- Normalized mass distributions of hydrogen (black), helium (magenta), carbon (green), oxygen plus neon and magnesium (red), and "nickel" (including iron-group elements and silicon; blue) versus radial velocity $v_{r}$ for the 3D simulation (right) and a 2D simulation performed for the meridional slice of the 3D model indicated in Fig. 5] (left). From top to bottom, the distributions are given at about 350s, $1000 \mathrm{~s}, 2600 \mathrm{~s}$, and $9000 \mathrm{~s}$ after core bounce. The binning is done in intervals of $\Delta v_{r}=100 \mathrm{~km} \mathrm{~s}^{-1}$ and the distributions $\Delta M_{i} / M_{i}$ with $i$ being the element index are given per unit length of velocity. Note the large differences between the $3 \mathrm{D}$ and $2 \mathrm{D}$ results of the $\mathrm{O}$ and $\mathrm{Ni}$ distributions at high velocities and of the hydrogen distribution at low velocities. 

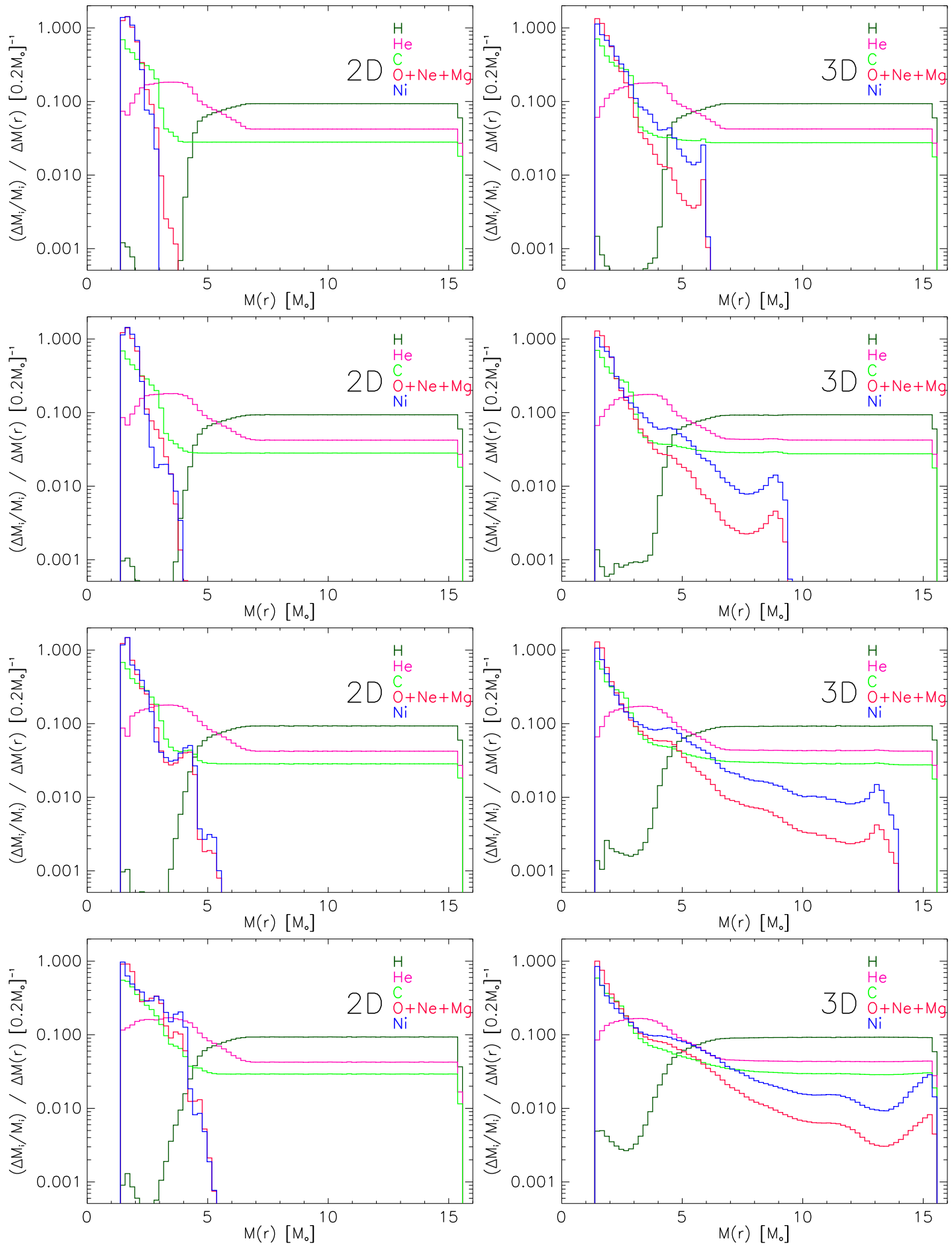

FIG. 7.- Normalized mass distributions of hydrogen (black), helium (magenta), carbon (green), oxygen plus neon and magnesium (red), and "nickel" (including iron-group elements and silicon; blue) versus enclosed mass $M(r)$ for the 3D simulation (right) and a 2D simulation performed for the meridional slice of the 3D model indicated in Fig. 5 (left). From top to bottom, the distributions are given at about $350 \mathrm{~s}, 1000 \mathrm{~s}, 2600 \mathrm{~s}$, and $9000 \mathrm{~s}$ after core bounce. The binning is done in intervals of $\Delta M(r)=0.2 M_{\odot}$ and the distributions $\Delta M_{i} / M_{i}$ with $i$ being the element index are given per unit length of mass. Note that in 3D oxygen and nickel clumps are able to penetrate through the hydrogen envelope, while they are stuck in the helium layer in the 2D case. 

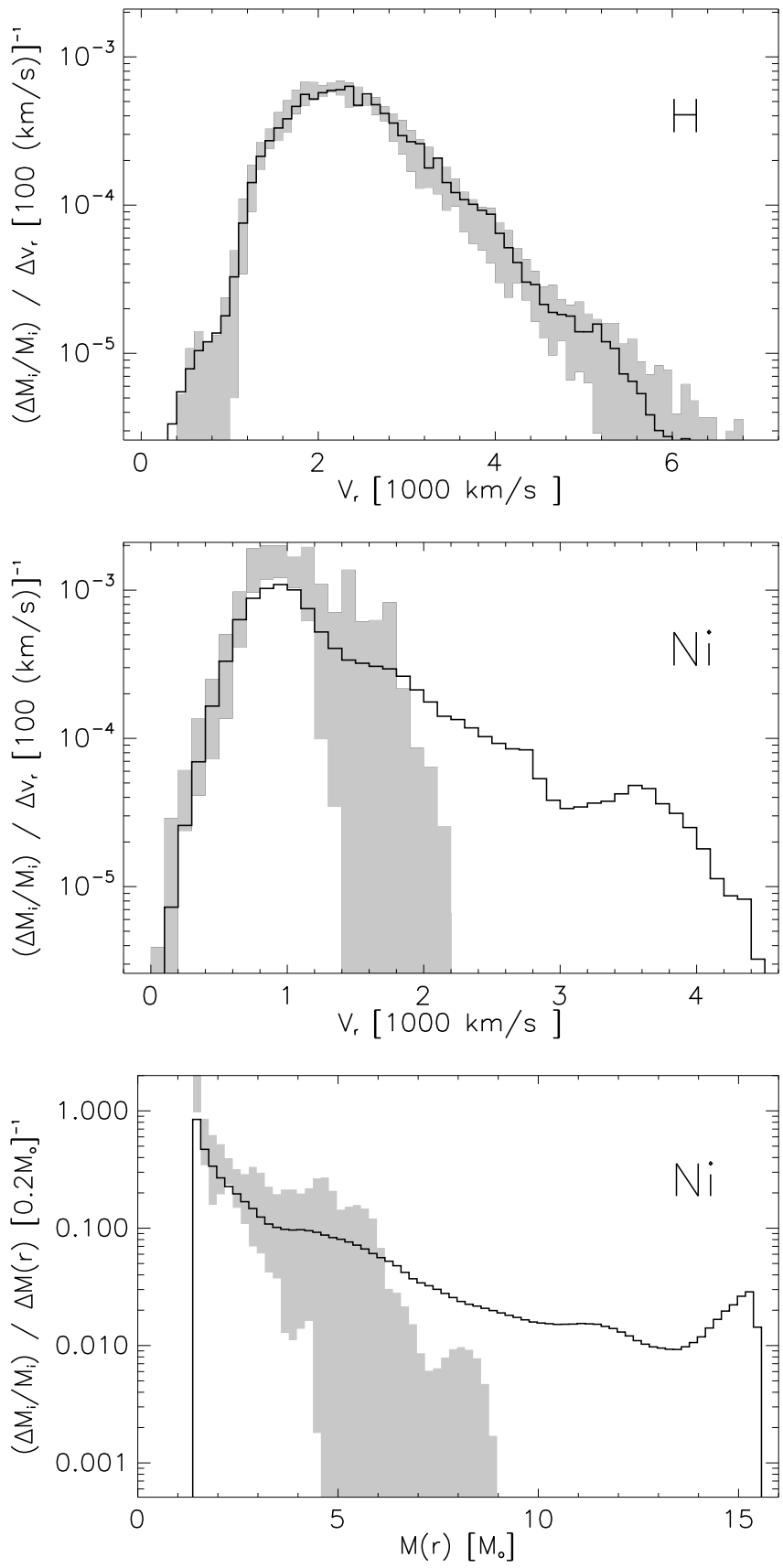

FIG. 8.- Normalized mass distributions of hydrogen (upper panel) versus radial velocity $v_{r}$ and of "nickel" (including iron-group elements and silicon) versus radial velocity $v_{r}$ (middle panel) as well as versus enclosed mass $M(r)$ (bottom panel). Results are shown for the 3D simulation (solid line) compared to the corresponding $2 \mathrm{D}$ results (grey region) at about $9000 \mathrm{~s}$. The binning is done in intervals of $\Delta v_{r}=100 \mathrm{~km} \mathrm{~s}^{-1}$ and of $\Delta M(r)=0.2 M \odot$, respectively, and the distributions $\Delta M_{i} / M_{i}$ with $i$ being the element index are given per unit length of velocity or mass, respectively. The grey-shaded area indicates the significant variation between the thirteen 2D simulations, which were started from different meridional slices of the initial 3D explosion model.

cases the metal-containing clumps thus get stuck in the dense helium wall as pointed out by Kifonidis et al. (2003). In contrast, in the 3D run nickel and oxygen are mixed deep into the hydrogen layer (and also a small amount of carbon is carried from the metal core into the hydrogen shell). The fastest nickel and oxygen clumps are able to penetrate nearly to the surface of the exploding star (in the mass space).

The two humps that can be discriminated in the mass distribution of these nuclear species well ahead of the bulk of the metal core in the mass and velocity space at $9000 \mathrm{~s}$, are linked to the few, but big and far-reaching structures, and to the greater number of smaller fingers that extend more uniformely to all directions in Fig. 2. The bigger bullets contain about three to four times more nickel than oxygen. They carry in total a mass of $\sim 2 \times 10^{-2} M_{\odot}$ with velocities of 3000 $4500 \mathrm{~km} \mathrm{~s}^{-1}$ for the nickel and $3000-4000 \mathrm{~km} \mathrm{~s}^{-1}$ for the oxygen. In contrast, the smaller finger-like structures move with $1500-3000 \mathrm{~km} \mathrm{~s}^{-1}$ and contain a total mass of $\sim 4 \times 10^{-2} M_{\odot}$.

It is amazing that in our 3D simulations the fastest of the metal clumps have achieved to overtake most of the hydrogen at the time the shock breaks out (at roughly $8000 \mathrm{~s}$ ). The nickel and oxygen mixing into the hydrogen envelope is clearly more efficient than even in the globally asymmetric explosions studied by Kifonidis et al. (2006), where at $10000 \mathrm{~s}$ the metals were seen to be distributed only up to an enclosed mass of about $10 M_{\odot}$ (see figure 6 there), although the peak metal velocities were roughly the same at $\sim 300$ s.

We refrain here from drawing far reaching conclusions on the observational consequences of this finding, e.g. concerning the early visibility of X-ray and $\gamma$-ray signatures of such strong mixing. While we consider the 2D/3D differences for the same explosion model and the same and fixed numerical resolution of our first explorative study as enlightening, we think that more simulations with different explosion energies, different progenitor stars, and in particular also with finer grid zoning are desirable before the results can be interpreted quantitatively with respect to observations of supernovae.

\section{A SIMPLE MODEL FOR CLUMP PROPAGATION}

In the following we will present a simple analysis that provides support for our hypothesis that the big discrepancies found in 3D compared to $2 \mathrm{D}$ models are to a large extent the consequence of geometry-dependent differences of the propagation and deceleration of objects ("clumps") under the influence of drag forces in the stellar medium of the helium layer.

\subsection{Time-independent toy model}

A first qualitative understanding can be obtained by considering objects moving in a stellar medium with a timeindependent density $\rho(r)$. We assume that the clump mass $m=\rho_{\mathrm{c}} V_{\mathrm{c}}$ remains constant and the volume $V_{\mathrm{c}}$ and density $\rho_{\mathrm{c}}$ of the clumps adjust to the local environmental conditions accordingly. With a velocity $v$ relative to the surrounding gas the equation of motion of the clumps at a radius $r$ can be written as

$$
m \frac{\mathrm{d} v(r)}{\mathrm{d} t}=\frac{1}{2} m \frac{\mathrm{d} v^{2}(r)}{\mathrm{d} r}=-F_{\mathrm{drag}}(r),
$$

where the drag force for high Reynolds numbers $(\mathrm{Re}=$ $\rho v V_{\mathrm{c}}\left(A_{\mathrm{c}} \mu\right)^{-1} \gg 1$ with $\mu$ being the dynamical viscosity and $A_{\mathrm{c}}$ the area the object presents to the flow) is given by

$$
F_{\text {drag }}(r)=\frac{1}{2} C A_{\mathrm{c}}(r) \rho(r) v^{2}(r) .
$$

The drag coefficient $C$ is a dimensionless parameter of order unity, which depends on the shape and the surface structure of the moving object. Equation (1) with Eq. (2) has an analytic 
solution for the velocity as a function of distance:

$$
\frac{v^{2}(r)}{v_{0}^{2}}=\exp \left(-\frac{C}{m} \int_{r_{0}}^{r} \mathrm{~d} r^{\prime} A_{\mathrm{c}}\left(r^{\prime}\right) \rho\left(r^{\prime}\right)\right) \equiv \mathrm{e}^{-2 f(r)},
$$

when $v_{0}$ is the initial velocity of the object at radial position $r_{0}$. The exponential decay of the initial velocity due to the influence of the drag force depends on the density of the medium through which the object propagates, on the drag coefficient, the mass of the object, and the area it presents to the flow.

In the following, we will discuss how the term in the exponent depends on the dimensionality of the simulation. Because of the assumed axial symmetry, in 2D all objects have a toroidal form. Let us consider in 2D an ideal torus with radius $r_{\mathrm{t}}$, whose center has a distance $r$ from the origin of the stellar grid and a distance $r \sin \theta$ from the symmetry axis, and compare its outward propagation in radial direction with that of a sphere with radius $r_{\mathrm{s}}$ in 3D also at radial distance $r$ from the center.

These objects, torus and sphere, are assumed to have a fixed shape but their size can vary as they move in radial direction from the initial position $r_{0}$ to $r$. In the beginning, the torus radius is $r_{\mathrm{t}, 0}$, and the spherical blob has a radius $r_{\mathrm{s}, 0}$. In order to compare the propagation of structures in $3 \mathrm{D}$ and $2 \mathrm{D}$ as in the hydrodynamical simulations, we suppose that the torus and sphere have initially the same cross section and thus radius: $r_{\mathrm{s}, 0}=r_{\mathrm{t}, 0}$. Since we assume the same density inside the objects, $\rho_{\mathrm{t}, 0}=\rho_{\mathrm{s}, 0}$, but the torus volume $V_{\mathrm{t}, 0}=2 \pi^{2} r_{\mathrm{t}, 0}^{2} r_{0} \sin \theta$ in general differs from the volume of the sphere, $V_{\mathrm{s}, 0}=\frac{4 \pi}{3} r_{\mathrm{s}, 0}^{3}$, the masses of torus and sphere are also unequal: $m_{\mathrm{t}} \neq m_{\mathrm{s}}$. Besides these different masses, the two objects also present different areas to the flow, $A_{\mathrm{t}, 0}=4 \pi r_{\mathrm{t}, 0} r_{0} \sin \theta$ and $A_{\mathrm{s}, 0}=\pi r_{\mathrm{s}, 0}^{2}$.

Moving in radial direction, the clumps are assumed to maintain pressure equilibrium with the surrounding medium. As a consequence, their density and therefore their volume and cross section will change. For the torus one has the scaling relations $V_{\mathrm{t}}=V_{\mathrm{t}, 0}\left(r_{\mathrm{t}} / r_{\mathrm{t}, 0}\right)^{2}\left(r / r_{0}\right)$ and $A_{\mathrm{t}}=A_{\mathrm{t}, 0}\left(r_{\mathrm{t}} / r_{\mathrm{t}, 0}\right)\left(r / r_{0}\right)$, while for the sphere they are $V_{\mathrm{s}}=V_{\mathrm{s}, 0}\left(r_{\mathrm{s}} / r_{\mathrm{s}, 0}\right)^{3}$ and $A_{\mathrm{s}}=$ $A_{\mathrm{s}, 0}\left(r_{\mathrm{s}} / r_{\mathrm{s}, 0}\right)^{2}$. Using this and the equality of the initial density and radii of torus and sphere, the arguments in the exponential function of Eq. (3) can be compared for the two geometries to yield the ratio:

$\frac{f_{\mathrm{t}}(r)}{f_{\mathrm{s}}(r)}=\frac{C_{\mathrm{t}}}{C_{\mathrm{s}}} \frac{8}{3 \pi}\left[\int_{r_{0}}^{r} \mathrm{~d} r^{\prime} \rho\left(r^{\prime}\right) \frac{r_{\mathrm{t}}}{r_{\mathrm{t}, 0}} \frac{r^{\prime}}{r_{0}}\right]\left[\int_{r_{0}}^{r} \mathrm{~d} r^{\prime} \rho\left(r^{\prime}\right)\left(\frac{r_{\mathrm{s}}}{r_{\mathrm{s}, 0}}\right)^{2}\right]^{-1}$.

In this equation the radii of the torus, $r_{t}$, and of the sphere, $r_{\mathrm{s}}$, in general depend on the distance $r$ from the grid center, if the clump propagates through a stellar medium with a pressure gradient. It should be noted that the ratio $f_{\mathrm{t}}(r) / f_{\mathrm{s}}(r)$ is nearly independent of both the mass difference and the initial difference of the torus and sphere areas perpendicular to the radial direction (the numerical factor $8 /(3 \pi)$ is very close to unity). Instead, the deceleration of the motion by drag forces depends crucially on the evolution of the areas as reflected by the radius-dependent functions in the integrands.

We assume that the pressure in the clump (torus or sphere) scales with the density in the clump as $P_{\mathrm{c}} \propto \rho_{\mathrm{c}}^{m} \propto V_{\mathrm{c}}^{-m}$, and that the pressure in the stellar environment follows a radial profile $P(r) \propto r^{-n}$. Using this and the requirement of pressure equilibrium, we find that the radii of torus and sphere change with $r$ according to

$$
\frac{r_{\mathrm{t}}}{r_{\mathrm{t}, 0}}=\left(\frac{r}{r_{0}}\right)^{\frac{1}{2} \frac{n}{m}-\frac{1}{2}}, \quad \frac{r_{\mathrm{s}}}{r_{\mathrm{s}, 0}}=\left(\frac{r}{r_{0}}\right)^{\frac{n}{3 m}} .
$$

Plugging Eq. (5) into Eq. (4), we obtain

$$
\begin{aligned}
\frac{f_{\mathrm{t}}(r)}{f_{\mathrm{s}}(r)}=\frac{C_{\mathrm{t}}}{C_{\mathrm{s}}} \frac{8}{3 \pi} & {\left[\int_{r_{0}}^{r} \mathrm{~d} r^{\prime} \rho\left(r^{\prime}\right)\left(\frac{r^{\prime}}{r_{0}}\right)^{\frac{1}{2} \frac{n}{m}+\frac{1}{2}}\right] } \\
\times & {\left[\int_{r_{0}}^{r} \mathrm{~d} r^{\prime} \rho\left(r^{\prime}\right)\left(\frac{r^{\prime}}{r_{0}}\right)^{\frac{2 n}{3 m}}\right]^{-1} . }
\end{aligned}
$$

The torus area perpendicular to the radial direction grows faster than that of the sphere if $n<3 m$, in which case the integral in the numerator increases more rapidly with $r$ than the one in the denominator. This difference is a consequence of the fact that any radial motion leads to a larger distance of the torus from the polar axis of the grid. This stretches the torus and causes an adjustment of its cross section according to pressure equilibrium. Since in addition to the area effect also the drag coefficient of the toroidal object is likely to be larger than that of the sphere, $C_{\mathrm{t}} \gtrsim C_{\mathrm{s}}$, the motion of the torus is clearly more strongly damped by the drag than that of the sphere if $n<3 m$.

In the supernova the clumps propagate through the medium of the dense shell of shock-accelerated progenitor matter. In this shell the density and pressure gradients are nearly flat, i.e., $\rho \sim$ const and $n \sim 0$ (see, e.g., Nomoto et al. (1994), figs. 31 and 32). This case is particularly suitable to illustrate the inadequacy of axisymmetric toroidal clumps in 2D instead of spherical clumps in 3D. While the latter propagate through the homogeneous medium without any change of their area perpendicular to the direction of the motion, the tori are unavoidably stretched as they reach larger radii $r$, which leads to an increase of the cross-sectional area they present to the flow. Equation (6) yields:

$$
\frac{f_{\mathrm{t}}(r)}{f_{\mathrm{s}}(r)}\left(\frac{C_{\mathrm{t}}}{C_{\mathrm{s}}} \frac{8}{3 \pi}\right)^{-1}=\frac{2}{3}\left[\left(\frac{r}{r_{0}}\right)^{\frac{3}{2}}-1\right]\left[\frac{r}{r_{0}}-1\right]^{-1},
$$

which is larger than unity for $r>r_{0}$. A stronger deceleration of the toroidal clump is thus caused by the artificial constraints associated with the axisymmetry of all structures in the 2D geometry.

\subsection{Time-dependent toy model}

In a second step we intend to obtain a more quantitative insight by considering the clump propagation in the dense helium layer that forms after the passage of the outgoing shock through the $\mathrm{He} / \mathrm{H}$ interface (for details, see Kifonidis et al. 2003). The interaction with this shell was identified as the main reason why metal-carrying rising Rayleigh-Taylor structures were unable to penetrate into the $\mathrm{H}$-shell in the $2 \mathrm{D}$ simulations of Kifonidis et al. (2003). Here we will integrate the equation of motion for spherical clumps decelerated by the drag force in the 3D environment (in contrast to the 3D case of a spherical clump, the equation of motion of a 2D toroidal object defies an analytic solution due to the explicit dependence of the clump volume and cross-sectional area on the radial coordinate position $r$ ).

We assume that the He layer is homogeneous but has a time-dependent density $\rho(t)$. This is an acceptable simplification because numerical simulations show that the radial 
density variations in this layer are much smaller than the timedependent decrease of the density due to the expansion of the star during the first three hours of the explosion. In the 3D case this leaves us just with a time-dependence of the drag force, $F_{\text {drag }}=F_{\text {drag }}(t)$, and thus

$$
m \frac{\mathrm{d} v(r)}{\mathrm{d} t}=-\frac{1}{2} C_{\mathrm{s}} A_{\mathrm{s}}(t) \rho(t) v^{2}(t) .
$$

Again we consider $m=$ const and $C_{\mathrm{s}}=$ const and $v(t)$ as the clump velocity relative to the surrounding medium. We further assume that the density of the helium layer evolves like $\rho(t)=\rho_{0}\left(t_{0} / t\right)^{k}$ and the pressure and density are linked by $P(t)=K \rho^{\gamma}(t)$. Using the same pressure-density relation for the medium inside the clumps, $P_{\mathrm{s}}=K_{\mathrm{s}} \rho_{\mathrm{s}}^{\gamma}$, but with a different constant $K_{\mathrm{s}} \neq K$ (corresponding to an entropy of the clumps different from that of the surroundings), the request of pressure equilibrium between clumps and environment allows us to calculate the time evolution of the clump radius,

$$
r_{\mathrm{s}}(t)=r_{\mathrm{s}, 0}\left(\frac{K_{\mathrm{s}}}{K}\right)^{\frac{1}{3 \gamma}}\left(\frac{\rho_{\mathrm{s}, 0}}{\rho_{0}}\right)^{\frac{1}{3}}\left(\frac{t}{t_{0}}\right)^{\frac{k}{3}}
$$

and therefore the time-dependent cross-sectional area that the clumps present to the surrounding medium: $A_{\mathrm{s}}(t)=$ $A_{\mathrm{s}, 0}\left(r / r_{\mathrm{s}, 0}\right)^{2} \equiv A^{*}\left(t / t_{0}\right)^{2 k / 3}$ with constant $A^{*}$. Inserting this and $\rho(t)$ into $\mathrm{Eq}(8)$, we can integrate the resulting differential equation by separation of variables. This yields the time evolution of the velocity (for $k \neq 3$ ) as

$$
v(t)=v_{0}\left\{1+\frac{C_{\mathrm{s}} A^{*}}{2 m} v_{0} t_{0} \rho_{0} \frac{1}{1-k / 3}\left[\left(\frac{t}{t_{0}}\right)^{1-k / 3}-1\right]\right\}^{-1} .
$$

We specialize now to $k=2$ and $\gamma=\frac{4}{2}$, which is in reasonable agreement with the simulations (Kifonidis et al. 2003). Rewriting the constant factor of the second term in the denominator by using the expressions for clump area and volume, we end up with

$v(t)=v_{0}\left\{1+\frac{9}{8}\left(\frac{4 \pi}{3}\right)^{\frac{1}{3}}\left(\frac{K_{\mathrm{s}}}{K}\right)^{\frac{1}{2}} C_{\mathrm{s}} v_{0} t_{0}\left(\frac{\rho_{0}}{m}\right)^{\frac{1}{3}}\left[\left(\frac{t}{t_{0}}\right)^{\frac{1}{3}}-1\right]\right\}^{-1}$.

The fastest clumps, which are also the most massive ones with $m \sim 4 \times 10^{-3} M_{\odot}$ in our 3D simulations, enter the dense helium shell with a velocity of $v_{0} \sim 2000-4000 \mathrm{~km} \mathrm{~s}^{-1}$ (relative to the ambient medium) at $t_{0} \sim 300 \mathrm{~s}$. At this time the density of the medium in the shell is $\rho_{0} \sim 0.1 \mathrm{~g} \mathrm{~cm}^{-3}$. The clumps typically have roughly three times lower entropy than the medium in the helium shell so that $K_{\mathrm{s}} / K \sim 1 / 3$ (most of the numerical values used here can be verified by closely inspecting the plots in Kifonidis et al. (2003)). Adopting for the drag coefficient plausible number 6 of $C_{\mathrm{s}} \sim 0.05 \ldots 0.2$, we obtain at $t=9000$ s velocities of $v \sim 1200-3100 \mathrm{~km} \mathrm{~s}^{-1}$ for the clump motion through the helium material, corresponding to a deceleration by roughly a factor of 2 . This means that the fastest objects are expected to still speed with $\sim 2400-4400 \mathrm{~km} \mathrm{~s}^{-1}$ relative to an observer in the lab frame. This is in good agreement with our hydrodynamical simulations and can thus be interpreted as support of our picture of the clump propaga-

\footnotetext{
${ }^{6}$ Unfortunately, no information is available in the literature for numbers that apply to the problem considered here.
}

tion under the influence of the drag force 7 . In contrast, in 2D models the maximum clump velocities are reduced to slightly more than $1500 \mathrm{kms}^{-1}$ in the lab frame (see Figs. 6, 8 and Kifonidis et al. (2003)), which is equal to the expansion velocity of the He-shell matter so that the clumps get stuck in their surroundings and are unable to penetrate into the hydrogen envelope (Fig. 7).

\section{SUMMARY AND CONCLUSIONS}

We have presented the first 3D simulations that followed the development of mixing instabilities in a supernova over a timescale of hours after the explosion was initiated by neutrino energy deposition. The considered progenitor was a $15.5 M_{\odot}$ blue supergiant star, which had also been adopted for the works of Kifonidis et al. (2003, 2006). Our long-time runs were started at roughly $0.5 \mathrm{~s}$ after core bounce from $3 \mathrm{D}$ models provided by Scheck (2007), who triggered the blast by suitably chosen neutrino luminosities imposed at the retreating inner grid boundary that replaced the high-density core of the nascent, contracting neutron star. The supernova runs we focussed our discussion on had an explosion energy of $10^{51}$ ergs. Our particular goal was a close comparison of the growth of mixing instabilities at the composition interfaces of the exploding star in 3D and 2D calculations. For the latter, we investigated a set of selected meridional slices of the initial 3D explosion models.

Our comparison revealed that the asymptotic velocities of metal-rich clumps are much higher in 3D than in the corresponding $2 \mathrm{D}$ cases. In the latter the iron-group and oxygen carrying dense fingers get stuck in the massive, dense helium shell ("wall") that forms below the base of the hydrogen envelope, and stay comoving with the helium matter there at a velocity of $\lesssim 1500 \mathrm{~km} \mathrm{~s}^{-1}$, a fact that was pointed out by Kifonidis et al. (2003), whose results are in agreement with our $2 \mathrm{D}$ simulations. In contrast, in the $3 \mathrm{D}$ runs we obtained "nickel" (三elements of the iron group plus silicon) ${ }^{8}$ and oxygen (plus neon and magnesium) bullets propagating at maximum velocities of $4500 \mathrm{~km} \mathrm{~s}^{-1}$ and a large fraction $(\sim 10$ $20 \%$ ) of the metal core of the progenitor star was seen to reach velocities of more than $2000 \mathrm{~km} \mathrm{~s}^{-1}$. The most extended and fastest Rayleigh-Taylor structures, some of which have a mass of several $10^{-3} M_{\odot}$, were found to contain mostly nickel. These nickel "bullets" expand significantly more rapidly than the longest fingers with dominant or appreciable oxygen content, which are smaller on average but much more numerous than the large nickel features. Iron-group nuclei, neon, and magnesium are thus carried far into the hydrogen layer. They move well ahead of oxygen-rich knots and both iron and oxygen overtake the material from the carbon layer in the ejecta. The onion-shell stratification of the progenitor is thus partially turned over during the explosion.

Besides strong mixing of heavy-elements into the hydrogen envelope, we also found about $0.086 M_{\odot}$ of hydrogen being transported deep into the metal core of the star to regions with velocities of $<1000 \mathrm{~km} \mathrm{~s}^{-1}, \sim 0.034 M_{\odot}$ to an enclosed mass of $M(r)<2 M_{\odot}$, and $\sim 0.19 M_{\odot}$ to $M(r)<4 M_{\odot}$. Although

\footnotetext{
${ }^{7}$ Note that the numerical result for the final velocity varies relatively little when different combinations of values for $v_{0}, \rho_{0}$, and $t_{0}$ within reasonable ranges are adopted.

${ }^{8}$ We note again that in our present models without nuclear burning a small region of silicon in the collapsing stellar core was lumped together with the iron-group nuclei to what we called "nickel". Silicon was therefore not traced separately as a nucleosynthetic constituent of the ejecta.
} 
this result seems to be roughly compatible with observational constraints based on the lightcurve shape of SN 1987A (see Blinnikov 1999 and Kifonidis et al. 2006), we refrain from a more detailed comparison and too far reaching conclusions, because neither the considered progenitor star is very suitable to compare with SN 1987A, nor were the explosion parameters (mass cut, nickel mass, explosion energy) adjusted to be optimally consistent with the observations of this supernova. Moreover, the exact amount of inward hydrogen mixing must be expected to be sensitive to the numerical resolution and thus at least convergence tests with better spatial zoning are required.

Kifonidis et al. (2006) obtained similar results on the basis of 2D explosion models where a supernova shock with a large global asphericity triggered strong Richtmyer-Meshkov instability at its passage through the composition interfaces, in particular also at the helium/hydrogen boundary. Such an effect, however, does not play any significant role in the present 3D simulations, because the shock starts out with much less initial deformation and is close to spherical when it crosses from the He into the $\mathrm{H}$ shell. Nevertheless, the peak velocities of the metals in our 3D simulations are even higher than those found by Kifonidis et al. (2006), and correspondingly the fastest nickel and oxygen clumps are able to penetrate even farther into the hydrogen envelope.

A closer comparison showed that a small fraction of the metal core receives velocities of more than $6000 \mathrm{~km} \mathrm{~s}^{-1}$ initially, which is significantly higher than in our 2D runs (but similar to the peak metal velocities seen by Kifonidis et al. (2006)). These clumps penetrate into the hydrogen envelope faster than the helium wall builds up. They achieve to retain a speed of more than $4000 \mathrm{~km} \mathrm{~s}^{-1}$ at the time of shock breakout and experience much less deceleration than in the 2D models of Kifonidis et al. (2006). Moreover, a larger fraction of the core metals start out with velocities of about $4000 \mathrm{~km} \mathrm{~s}^{-1}$, which is of the order of the speed of the fastest clumps in our 2D models, but again the deceleration of these dense bullets is less strong than in the $2 \mathrm{D}$ case. This suggests that the final differences can be attributed only partly to 2D/3D differences in the growth rate of perturbations at the composition interfaces, which were pointed out by Kane et al. (2000). Instead, the discrepancies between 3D and 2D results that develop when the majority of the metal-rich clumps begins to enter the dense helium wall below the $\mathrm{He} / \mathrm{H}$ boundary after $\sim 1000 \mathrm{~s}$, and the increase of these discrepancies over the first 1-2 hours after the onset of the explosion while the clunps make their way through the helium layer, require a different explanation.

We therefore hypothesized that these differences are a consequence of differences in the drag forces of the surrounding medium, which affect the propagation of the metal-rich clumps and lead to their deceleration as they plow through the helium layer. By means of a simple analytic model we could demonstrate that the different geometry of the bullets in 2D and 3D - toroidal (axisymmetric) structures versus quasi-spherical bubbles - can explain the differences observed in our simulations. Making reasonable assumptions in a toy model description of the conditions in the exploding star and using plausible values of the drag coefficient, we could come up with numbers that are in the ballpark of the asymptotic nickel clump velocities in the hydrogen shell found in our 3D hydrodynamic models.

In this context experimentally and numerically established data for the drag coefficient in dependence of the clump ge- ometry would have been highly desirable but could not be found in the literature. A determination of corresponding values may be a valuable goal for future investigations, e.g. at the National Ignition Facility (NIF) at Livermore. It must be suspected that the drag force acting on the dense metal clumps is not independent of the grid resolution and insufficient zoning could lead to an underestimation of drag effects on the clump propagation. Without applying any adaptive mesh refinement (AMR) techniques, the 1200 radial zones and $1^{\circ}$ resolution in azimuthal and $0.935^{\circ}$ in lateral direction were the best we could allow for as a compromise between accuracy and CPUtime requirements. Although our $2 \mathrm{D}$ results are in reasonably good agreement with the 2D AMR calculations performed by Kifonidis et al. (2003, 2006), we still think that simulations with varied mesh size or an AMR code would be useful to confirm our findings in 3D.

In this pilot study we were aiming at a first analysis of fundamental differences between mixing instabilities during the long-time evolution of supernova explosions in two and three dimensions rather than attempting to match the observational appearance of special events like, e.g., SN 1987A (although we used this supernova as the best studied one for our motivation and also referred to it when our findings agreed with basic features that SN 1987A is likely to have in common with a wider class of cases). On the basis of our results, which appear suitable to account for the strong inward mixing of hydrogen and outward mixing of metals that is needed to explain the smoothness of the light curve as well as the early appearance of X-ray and gamma-ray emission of SN 1987A, we therefore do not conclude that all observational properties of this supernova can be reproduced by a relatively weakly aspherical initiation of the explosion. More simulations are needed to clarify this point, in particular by employing a stellar model that is compatible with all knowledge acquired about the progenitor structure of SN 1987A during the past 20 years.

While we think that the 2D/3D differences found in our investigation are likely to be generic, the extent of the radial mixing, the size and mass distribution of the metal fingers, their velocities and spatial distribution, and the composition of individual clumps must be expected to depend strongly on the structure of the progenitor star, the explosion energy, and the initial asymmetry of the blast. Having advanced the modeling of mixing instabilities in supernova explosions to the third dimension, we therefore plan to direct our focus to the exploration of a wider variety of progenitor stars with the goal to establish a link between explosion models and observations. Besides SN 1987A, the geometry of the Cassiopeia A remnant has been studied observationally in very much detail (e.g., Hwang et al. (2004); Fesen et al. (2006a b), and references therein). It exhibits properties such as the radial turnover of the metal core that are compatible with the findings of this work, and a directional asymmetry that cannot be accounted for by models constrained to axial symmetry. Therefore not only the differences of $3 \mathrm{D}$ versus $2 \mathrm{D}$ results reported in this paper but also observational properties of supernovae and supernova remnants point to the indispensability of modeling the explosions in three dimensions. An interesting question certainly is, whether the jet-like, silicon-rich features observed in Cas A could be morphologically linked to the structures seen as the fastest and most massive RayleighTaylor fingers in our simulations. Although our results appear suggestive in this respect, a convincing answer requires investigations of progenitors and explosion parameters fitting this 
remnant as well as a more complete consideration of the nuclear composition of the ejecta.

We thank L. Scheck for providing us with his 3D explosion models as initial data for our studies, V. Utrobin for sharing with us his insight into modelling observations of SN 1987A, and M. Rampp (Rechenzentrum Garching) for his great assistance in the visualization of our 3D data.
This work was supported by the Deutsche Forschungsgemeinschaft through the Transregional Collaborative Research Centers SFB/TR 27 "Neutrinos and Beyond" and SFB/TR 7 "Gravitational Wave Astronomy", the Collaborative Research Center SFB-375 "Astro-Particle Physics", and the Cluster of Excellence EXC 153 "Origin and Structure of the Universe" (http://www.universe-cluster.de). The computations were performed on the SGI Altix 4700 of the LeibnizRechenzentrum (LRZ) in Munich.

\section{REFERENCES}

Anuchina, N., Volkov, V., Gordeychuk, V., Eskov, N., Ilyutina, O., \& Kozyrev, O. 2004, J. Comp. Appl. Math., 168, 11

Arnett, W. D., Bahcall, J. N., Kirshner, R. P., \& Woosley, S. E. 1989, Annual Review of Astronomy and Astrophysics, 27, 629

Aschenbach, B., Egger, R., \& Trümper, J. 1995, Nature, 373, 587

Benz, W., \& Thielemann, F.-K. 1990, ApJ, 348, L17

Blair, W. P., et al. 2000, ApJ, 537, 667

Blinnikov, S. I. 1999, Astronomy Letters, 25, 359

Blinnikov, S., Lundqvist, P., Bartunov, O., Nomoto, K., \& Iwamoto, K. 2000, ApJ, 532, 1132

Blondin, J. M., \& Mezzacappa, A. 2007, Nature, 445, 58

Blondin, J. M., Mezzacappa, A., \& DeMarino, C. 2003, ApJ, 584, 971

Burrows, A., Livne, E., Dessart, L., Ott, C. D., \& Murphy, J. 2006, ApJ, 640,878

-. 2007, ApJ, 655, 416

Cabot, W. 2006, Physics of Fluids, 18, 045101

Colella, P. \& Glaz, H. H. 1984, J. Comput. Phys., 59, 264

Colella, P. \& Woodward, P. R. 1984, J.Comput.Phys., 54, 174

Couch, S. M., Wheeler, J. C., \& Milosavljević, M. 2009, ApJ, 696, 953

Fassia, A., \& Meikle, W. P. S. 1999, Mon. Not. R. Astron. Soc., 302, 314

Fassia, A., Meikle, W. P. S., Geballe, T. R., Walton, N. A., Pollacco, D. L.,

Rutten, R. G. M., \& Tinney, C. 1998, Mon. Not. R. Astron. Soc., 299, 150

Fesen, R. A., et al. 2006a, ApJ, 636, 859

-. 2006b, ApJ, 645, 283

Fryer, C. L., \& Warren, M. S. 2002, ApJ, 574, L65

Fryxell, B., Arnett, D., \& Müller, E. 1991, ApJ, 367, 619

Hachisu, I., Matsuda, T., Nomoto, K., \& Shigeyama, T. 1990, ApJ, 358, L57

-. 1992, ApJ, 390, 230

Hammer, N. J. 2009, PhD Thesis, Technische Universität München

Hanuschik, R. W., Thimm, G., \& Dachs, J. 1988, MNRAS, 234, 41P

Herant, M., \& Benz, W. 1991, ApJ, 370, L81

-. 1992, ApJ, 387, 294

Hirata, K., Kajita, T., Koshiba, M., Nakahata, M., \& Oyama, Y. 1987, Physical Review Letters, 58, 1490

Hughes, J. P., Rakowski, C. E., Burrows, D. N., \& Slane, P. O. 2000, ApJ, 528, L109

Hwang, U., et al. 2004, ApJ, 615, L117

Janka, H.-T., Langanke, K., Marek, A., Martínez-Pinedo, G., \& Müller, B. 2007, Phys. Rep., 442, 38

Joggerst, C. C., Woosley, S. E., \& Heger, A. 2009, ApJ, 693, 1780

Kane, J., Arnett, D., Remington, B. A., Glendinning, S. G., Bazán, G., Müller, E., Fryxell, B. A., \& Teyssier, R. 2000, ApJ, 528, 989

Kawabata, K. S., et al. 2002, ApJ, 580, L39

Kifonidis, K., Plewa, T., Janka, H.-T., \& Müller, E. 2003, Astron. Astrophys., 408, 621

Kifonidis, K., Plewa, T., Scheck, L., Janka, H.-T., \& Müller, E. 2006, Astron. Astrophys., 453, 661
Leonard, D. C., Filippenko, A. V., Ardila, D. R., \& Brotherton, M. S. 2001, ApJ, 553, 861

Leonard, D. C., et al. 2006, Nature, 440, 505

Li, H., McCray, R., \& Sunyaev, R. A. 1993, ApJ, 419, 824

Marek, A., \& Janka, H.-T. 2009, ApJ, 694, 664

Maund, J. R., Wheeler, J. C., Patat, F., Wang, L., Baade, D., \& Höflich, P. A. 2007, ApJ, 671, 1944

Mazzali, P. A., et al. 2007, ApJ, 670, 592

Miceli, M., Bocchino, F., \& Reale, F. 2008, ApJ, 676, 1064

Modjaz, M., Kirshner, R. P., Blondin, S., Challis, P., \& Matheson, T. 2008, ApJ, 687, L9

Müller, E., Fryxell, B., \& Arnett, D. 1991a, in European Southern Observatory Astrophysics Symposia, Vol. 37, European Southern Observatory Astrophysics Symposia, ed. I. J. Danziger \& K. Kjaer, 99

Müller, E., Fryxell, B., \& Arnett, D. 1991b, Astron. Astrophys., 251, 505

Müller, E., Hillebrandt, W., Orio, M., Hoflich, P., Monchmeyer, R., \& Fryxell, B. A. 1989, A\&A, 220, 167

Nagasawa, M., Nakamura, T., \& Miyama, S. M. 1988, PASJ, 40, 691

Nomoto, K., Shigeyama, T., Kumagai, S., Yamaoka, H., \& Suzuki, T. 1994, in Supernovae, ed. S. A. Bludman, R. Mochkovitch, \& J. Zinn-Justin, 489-+

Noro, A., Miyaji, S., Yamashita, K., Ogawa, T., \& den, M. 2004, in IAU Symposium, Vol. 218, Young Neutron Stars and Their Environments, ed. F. Camilo \& B. M. Gaensler, 37

Noro, A., Ogawa, T., Miyaji, S., Yamashita, K., \& den, M. 2002, in 8th Asian-Pacific Regional Meeting, Volume II, ed. S. Ikeuchi, J. Hearnshaw, \& T. Hanawa, 451-452

Plewa, T. \& Müller, E. 1999, A\&A, 342, 179

Remington, B. A., Drake, R. P., \& Ryutov, D. D. 2006, Reviews of Modern Physics, 78, 755

Scheck, L. 2007, PhD thesis, Technical University Munich

Scheck, L., Janka, H.-T., Foglizzo, T., \& Kifonidis, K. 2008, Astron. Astrophys., 477, 931

Scheck, L., Kifonidis, K., Janka, H.-T., \& Müller, E. 2006, Astron. Astrophys., 457, 963

Scheck, L., Plewa, T., Janka, H.-T., Kifonidis, K., \& Müller, E. 2004, Physical Review Letters, 92, 011103

Shigeyama, T., \& Nomoto, K. 1990, ApJ, 360, 242

Spyromilio, J., Meikle, W. P. S., \& Allen, D. A. 1990, MNRAS, 242, 669

Strang, G. 1968, SIAM J.Numer.Anal., 5, 506

Timmes, F. X., \& Swesty, F. D. 2000, ApJ, Suppl. Ser., 126, 501

Utrobin, V. P. 2004, Astronomy Letters, 30, 293

Utrobin, V. P., Chugai, N. N., \& Andronova, A. A. 1995, A\&A, 295, 129

Wang, L., Baade, D., Höflich, P., \& Wheeler, J. C. 2003, ApJ, 592, 457

Wang, L., \& Wheeler, J. C. 2008, ARA\&A, 46, 433

Woosley, S. E. 1988, ApJ, 330, 218

Woosley, S. E., Pinto, P. A., \& Ensman, L. 1988, ApJ, 324, 466

Yamada, Y., Nakamura, T., \& Oohara, K. 1990, Progress of Theoretical Physics, 84, 436 